\title{
ON NUMERICAL APPROXIMATION OF ATANGANA-BALEANU-CAPUTO FRACTIONAL INTEGRO-DIFFERENTIAL EQUATIONS UNDER UNCERTAINTY IN HILBERT SPACE
}

\author{
Mohammed Al-Smadi ${ }^{1,2}$, Hemen Dutta ${ }^{3, *}$, Shatha Hasan $^{1}$ \\ AND SHAHER MOMANI ${ }^{2,4}$
}

\begin{abstract}
Many dynamic systems can be modeled by fractional differential equations in which some external parameters occur under uncertainty. Although these parameters increase the complexity, they present more acceptable solutions. With the aid of Atangana-Baleanu-Caputo (ABC) fractional differential operator, an advanced numerical-analysis approach is considered and applied in this work to deal with different classes of fuzzy integrodifferential equations of fractional order fitted with uncertain constraints conditions. The fractional derivative of ABC is adopted under the generalized H-differentiability (g-HD) framework, which uses the Mittag-Leffler function as a nonlocal kernel to better describe the timescale of the fuzzy models. Towards this end, applications of reproducing kernel algorithm are extended to solve classes of linear and nonlinear fuzzy fractional ABC Volterra-Fredholm integrodifferential equations. Based on the characterization theorem, preconditions are established under the Lipschitz condition to characterize the fuzzy solution in a coupled equivalent system of crisp ABC integrodifferential equations. Parametric solutions of the $\mathrm{ABC}$ interval are provided in terms of rapidly convergent series in Sobolev spaces. Several examples of fuzzy ABC Volterra-Fredholm models are implemented in light of g-HD to demonstrate the feasibility and efficiency of the designed algorithm. Numerical and graphical representations of both classical Caputo and ABC fractional derivatives are presented to show the effect of the ABC derivative on the parametric solutions of the posed models. The achieved results reveal that the proposed method is systematic and suitable for dealing with the fuzzy fractional problems arising in physics, technology, and engineering in terms of the ABC fractional derivative.
\end{abstract}

Mathematics Subject Classification. 45B05, 47B32, 34A07, 34A08, $45 \mathrm{~L} 05$.

Received March 9, 2021. Accepted May 24, 2021.

Keywords and phrases: Reproducing kernel algorithm, Fredholm-Volterra equation, characterization theorem for fuzzy integrodifferential equation, generalized H-differentiability, Atangana-Baleanu-Caputo derivative.

1 Department of Applied Science, Ajloun College, Al-Balqa Applied University, Ajloun 26816, Jordan.

2 Nonlinear Dynamics Research Center (NDRC), Ajman University, Ajman, UAE.

3 Department of Mathematics, Gauhati University, Gawahati, 781014 Assam, India.

4 Department of Mathematics, Faculty of Science, The University of Jordan, Amman 11942, Jordan.

* Corresponding author: hemen_dutta08@rediffmail.com 


\section{INTRODUCTION}

Modeling processes of natural phenomena create perceptions and general impressions about the dynamic behavior of any physical system that may involve uncertain parameters that result from many factors such as measurement errors, estimates, expectations, and deficient data. In this direction, fuzzy set theory has been established to describe the uncertainty that appears in mathematical modeling. In 1965, the standard theory was originally introduced by Zadeh [55]. Afterwards, Dubois and Prade [29] proposed the fuzzy real numbers notions along with some of the essential characteristics as well. In a later separate work, Kandel and Byatt [41] introduced the concept of fuzzy differential equations. Anyhow, there are several suggestions for defining fuzzy derivative operators as well as studying fuzzy differential equations, including Seikkala, Goetschel-Voxman, Hukuhara (H-differentiability), Puri-Ralescu, and strongly generalized differentiable concepts [3, 27, 30, 33]. The most popular approaches employing standard, strong, or generalized H-differentiability [11, 24, 36].

On the other hand, the theory of fractional calculus is an interesting topic, not only among mathematicians but also among physicists and engineers for its great importance applications in many fields of engineering and sciences $[12,13,15,20,31,34,43]$. It has been investigated extensively for describing memory and hereditary for various physical and engineering applications similar to rheology, continuum mechanics, entropy, electromagnetic problems, thermodynamics and so forth $[2,7,19,21,25,37,42,46]$. Further, in the literature, there are many definitions of fractional derivatives, such as the concepts of Caputo, Erdelyi-Kober, Riemann-Liouville, Graunwald-Letnikov, Riesz, and Caputo-Fabrizio [17, 22, 39, 44, 49-54]. The most common are the concepts of Riemann-Liouville and Caputo which bring some privacy. Nevertheless, they involve a singular kernel function that may adversely affect a realistic understanding of real-world problems. Indeed, Caputo-Fabrizio concept proposed in [25] includes an exponential kernel function to describe variants and structures of different scales, which cannot be well formulated by standard local derivatives possessing single kernels such as RiemannLiouville and Caputo. In this orientation, Atangana and Baleanu proposed a novel concept as a generalization of the Caputo-Fabrizio derivative in light of the generalized Mittag-Leffler function $E_{\alpha}\left(-z^{\alpha}\right)=\sum_{n=0}^{\infty} \frac{(-t)^{\alpha n}}{\Gamma(n \alpha+1)}$ to construct the non-singular as well as nonlocal kernel [21].

As a result of studying the fuzzy theory for fractional calculus, the term of fractional fuzzy differential equations (FFDEs) has been established in 2010 [1]. In [5], it has been proposed the fractional generalization by means of $\mathrm{H}$-differentiable. Recently, the authors $[4,56]$ defined a novel operator of fractional derivatives based on Atangana-Baleanu-Caputo (ABC) in view of fuzzy valued function with form of parametric interval, called $\mathrm{ABC}$ gH-differentiability. In this paper, we intend to study the effect of ABC gH-differentiability on the solution of different types of fuzzy fractional integrodifferential equations (FFIDEs). More specifically, we consider the underlying Fredholm-Volterra FFIDE:

$$
\left\{\begin{array}{l}
\left(\underset{g H}{A B C} D_{a^{+}}^{\beta} \omega\right)(t)=f(t)+p(t) \omega(t)+\int_{a}^{b} H(t, \tau, \omega(\tau)) \mathrm{d} \tau+\int_{a}^{t} K(t, \tau, \omega(\tau)) \mathrm{d} \tau, \\
\omega(a)=\eta, a \geq 0
\end{array}\right.
$$

where $t \geq a, 0<\beta \leq 1, \eta$ is a fuzzy number, $f(t)$ is a continuous fuzzy-valued function, $p(t)$ is a continuous realvalued function with nonnegative or nonpositive values on $[a, b]$, and $\underset{g H}{A B C} D_{a^{+}}^{\beta}(\cdot)$ denotes the $\mathrm{ABC}$ gH-derivative of order $\beta$ in view of continuous kernel functions $H(t, \tau, \omega(\tau))$ and $K(t, \tau, \omega(\tau))$.

By and large, there are no conventional techniques for finding exact solutions for FFIDEs. Therefore, there is an urgent need for advanced numerical methods to obtain accurate approximate solutions to these equations. In this analysis, we modify a numerical method based on the reproducing kernel theory to obtain approximate solutions of equation (1.1). The current method has many positive advantages. For instance, but not limited to, it is reliable and accurate numerical results can be achieved easily, it can be applied directly without any further assumptions on the structure of specific physical problems, it is not affected by cumulative calculation errors, and it is universal in nature and has a high capacity for solving various nonlinear mathematical issues. Therefore, the RKHS method has received enough attention in the last decade [8-10, 14, 16, 28, 38]. 
Motivated by the aforementioned discussion, this numerical research aims to design a novel iterative algorithm to obtain solutions to fuzzy integrodifferential equations in terms of the new $\mathrm{ABC}$-fractional concept containing nonsingular and nonlocal kernel under gH-differentiability in addition to studying the effect of ABC-fractional derivative on these solutions. To begin with, several kernel functions are created to establish a complete orthogonal system in the Hilbert space. For this purpose, a linear bounded and invertible fractional operator is defined to extend analytical solutions over a dense and compact interval. Based on reproducing kernel property, the approximate solutions converge uniformly to the analytical solutions. Eventually, some numerical examples are presented to illustrate the reliability and efficiency of the suggested algorithm. This paper is organized as follows: In Section 2, some basic concepts related to fuzzy calculus and fractional calculus are presented. In Section 3, the FFIDE converted into a fractional system of integrodifferential equations. The procedures of RKHSM for solving the general form of both linear and nonlinear FFIDEs are discussed in Section 4. Some numerical examples are carried out in Section 5. This paper ends with a conclusion in Section 6.

\section{Preliminaries And mathematical CONCEPTS}

In this section, some necessary definitions and mathematical preliminaries of fuzzy calculus and fractional calculus are introduced. To this end, the main concepts used in this analysis are presented, namely, the strongly generalized differentiability, ABC gH-differentiability, and Riemann integrability. Anyhow, a fuzzy number is a generalization of a real number in the sense that it does not refer to a single value but rather to a set of possible values, each with a weight between 0 and 1 . This weight is called the membership function.

Definition 2.1. [40] A fuzzy number $\eta$ is a mapping $\eta: \mathbb{R} \rightarrow[0,1]$ that satisfies the following properties:

(1) $\eta$ is normal. That is, there is $\xi \in \mathbb{R}$ with $\eta(\xi)=1$.

(2) $\eta$ is fuzzy convex. That is, $\eta(\gamma \xi+(1-\gamma) \zeta) \geq \min (\eta(\xi), \eta(\zeta))$ for all $\xi, \zeta \in \mathbb{R}$ and $\gamma \in[0,1]$.

(3) $\eta$ is upper semi-continuous.

(4) The set $\overline{\{\xi \in \mathbb{R}: \eta(\xi)>0\}}$ is bounded.

The set of all fuzzy numbers is denoted by $\mathbb{R}_{\mathcal{F}}$. An effective way to present a fuzzy number $\eta$ is by using its $r$-cut representation which is given by $[\eta]^{r}=\{x \in \mathbb{R}: \eta(x) \geq r\}$ for $r \in(0,1]$ and $[\eta]^{0}=\overline{\{x \in \mathbb{R}: \eta(x)>0\}}$. $[\eta]^{0}$ is called the support of $A$. The core of $A$ is the crisp set of all points $x$ in $\mathbb{R}$ such that $\eta(x)=1$. Obviously, if $\eta$ is a fuzzy number, then $[\eta]^{r}=\left[\eta_{1}(r), \eta_{2}(r)\right]$, where $\eta_{1}(r)=\eta_{1 r}=\min \left\{x \mid x \in[\eta]^{r}\right\}$ and $\eta_{2}(r)=\eta_{2 r}=$ $\max \left\{x \mid x \in[\eta]^{r}\right\} \quad \forall r \in[0,1]$. A common form of fuzzy numbers is the triangular fuzzy number, which is characterized below.

Definition 2.2. [45] A fuzzy number $\eta$ is said to be a generalized triangular fuzzy number, which is denoted by $(a, b, c ; \omega)$, if its membership function has the form:

$$
\eta(x)= \begin{cases}\frac{x-a}{b-a} \omega, & \text { if } a \leq x<b, \\ \frac{c-x}{c-b} \omega, & \text { if } b \leq x \leq c, \\ 0 & \text { otherwise }\end{cases}
$$

where $a, b, c \in \mathbb{R}$ and $a<b<c$.

The $r$-cut representation for a generalized triangular fuzzy number $(a, b, c ; \omega)$ is $[\eta]^{r}=\left[a+\frac{(b-a) r}{\omega}, c-\frac{(c-b) r}{\omega}\right]$ for any $r \in(0,1)$. For more illustration, the underlying theorem gives the necessary conditions of any two real valued functions $\eta_{1}$ and $\eta_{2}$ defined on $[0,1]$ to get the parametric form $\left[\eta_{1}(r), \eta_{2}(r)\right]$ of a fuzzy number $\eta$ for each $r \in[0,1]$. 
Theorem 2.3. [33] Suppose that $\eta_{1}, \eta_{2}:[0,1] \rightarrow \mathbb{R}$ satisfy the conditions; First, $\eta_{1}$ is a bounded monotonic nondecreasing left continuous function $\forall r \in(0,1]$ and right continuous for $r=0$; Second, $\eta_{2}$ is a bounded monotonic nonincreasing left continuous function $\forall r \in(0,1]$ and right continuous for $r=0$; Third, $\eta_{1}(1) \leq \eta_{2}(1)$. Then, $\eta: \mathbb{R} \rightarrow[0,1]$ which is defined by $\eta(x)=\operatorname{Sup}\left\{r \mid \eta_{1}(r) \leq x \leq \eta_{2}(r)\right\}$ is a fuzzy number with parameterization $[\eta]^{r}=\left[\eta_{1}(r), \eta_{2}(r)\right]$. Moreover, if $\eta$ is a fuzzy number with $[\eta]^{r}=\left[\eta_{1}(r), \eta_{2}(r)\right]$ (or simply, $\left.\left[\eta_{1 r}, \eta_{2 r}\right]\right)$, then the functions $\eta_{1}, \eta_{2}:[0,1] \rightarrow \mathbb{R}$ satisfy the above conditions. In this case, we can represent a fuzzy number by an ordered pair of functions $\left(\eta_{1}, \eta_{2}\right)$.

Arithmetic operations in $\mathbb{R}_{\mathcal{F}}$ can be defined as those on intervals of $\mathbb{R}$. So, for any $\gamma \in \mathbb{R} /\{0\}$, and $u, v \in$ $\mathbb{R}_{\mathcal{F}}$ with $[u]^{r}=\left[u_{1 r}, u_{2 r}\right]$ and $[v]^{r}=\left[v_{1 r}, v_{2 r}\right]$, we have $[u+v]^{r}=[u]^{r}+[v]^{r}=\left[u_{1 r}+v_{1 r}, u_{2 r}+v_{2 r}\right]$, and $[\gamma u]^{r}=\gamma[u]^{r}=\left[\min \left\{\gamma u_{1 r}, \gamma u_{2 r}\right\}, \max \left\{\gamma u_{1 r}, \gamma u_{2 r}\right\}\right]$. Obviously, $\mathbb{R}_{\mathcal{F}}$ does not form a vector space with the zero element $\{0\}$. Hence, additive simplification is not valid, that is $u+v=u+w \nRightarrow v=w$ for fuzzy numbers $u, v$ and $w$.

To overcome this situation, we have the Hukuhara difference (H-difference). The H-difference of $u, v \in \mathbb{R}_{\mathcal{F}}$, denoted by $u \ominus v=w$, is the fuzzy number that satisfies $u=v+w$. Its $r$-cut representation is $[u \ominus v]^{r}=$ $\left[u_{1 r}-v_{1 r}, u_{2 r}-v_{2 r}\right]$. In [24], Bede and Stefanini introduced the gH-difference as follows

$$
u \ominus_{g H} v=w \Leftrightarrow u=v+w \text { or } v=u+(-1) w
$$

with r-cuts $\left[u \ominus_{g H} v\right]^{r}=\left[\min \left\{u_{1 r}-v_{1 r}, u_{2 r}-v_{2 r}\right\}, \max \left\{u_{1 r}-v_{1 r}, u_{2 r}-v_{2 r}\right\}\right]$.

Definition 2.4. [47] The Housdorff metric $D$ on $\mathbb{R}_{\mathcal{F}}$ is defined by $D: \mathbb{R}_{\mathcal{F}} \times \mathbb{R}_{\mathcal{F}} \rightarrow \mathbb{R}^{+} \bigcup\{0\}$ such that $D(u, v)=$ $\operatorname{Sup}_{r \in[0,1]} \max \left\{\left|u_{1 r}-v_{1 r}\right|,\left|u_{2 r}-v_{2 r}\right|\right\}$ for any fuzzy numbers $u=\left(u_{1}, u_{2}\right)$ and $v=\left(v_{1}, v_{2}\right)$.

A fuzzy function on an interval $T$ is a mapping $F: T \rightarrow \mathbb{R}_{\mathcal{F}}$. If for fixed $t_{0} \in T$ and $\varepsilon>0, \exists \delta>0$ such that $\left|t-t_{0}\right|<\delta \Longrightarrow D\left(F(t), F\left(t_{0}\right)\right)<\varepsilon$, then we say that $F$ is continuous at $t_{0}$. If $F$ is continuous $\forall t \in T$, then $F$ is continuous on $T$ [32]. The differentiability of a fuzzy function can be defined as follows.

Definition 2.5. [4] Let $F:(a, b) \rightarrow \mathbb{R}_{\mathcal{F}}$ and $t_{0} \in(a, b)$. We say that $F$ is generalized Hukuhara differentiable (gH-differentiable) at $t_{0}$ if there exists a fuzzy number ${ }_{g H} D F\left(t_{0}\right)$ such that

$$
{ }_{g H} D F\left(t_{0}\right)=\lim _{h \rightarrow 0^{+}} \frac{F\left(t_{0}+h\right) \ominus_{g H} F\left(t_{0}\right)}{h}=\lim _{h \rightarrow 0^{+}} \frac{F\left(t_{0}\right) \ominus_{g H} F\left(t_{0}+h\right)}{h},
$$

where the limits here are taken in the metric space $\left(\mathbb{R}_{\mathcal{F}}, \mathrm{D}\right)$.

With the help of gH-difference (2.1), the parametric interval form of the gH-derivative for a function $F(t)$ has only one of the following forms:

- Case 1: (1)-differentiability: $\left[{ }_{1-g H} D F(t)\right]^{r}=\left[F_{1 r}^{\prime}(t), F_{2 r}^{\prime}(t)\right], \quad 0 \leq r \leq 1$.

- Case 2: (2)-differentiability: $\left[{ }_{2-g H} D F(t)\right]^{r}=\left[F_{2 r}^{\prime}(t), F_{1 r}^{\prime}(t)\right], 0 \leq r \leq 1$.

For integration of a fuzzy valued function, many approaches have been proposed such as Lebesgue integral [40] and fuzzy Riemann integral [6]. The Lebesgue integral ${ }_{g H} D F(t)$ within interval parametric form is more convenient, which is defined level wise by

$$
\left[\int_{a}^{t}{ }_{g H} D F(t) \mathrm{d} x\right]^{r}= \begin{cases}{\left[\int_{a}^{t} F_{1 r}^{\prime}(x) \mathrm{d} x, \int_{a}^{t} F_{2 r}^{\prime}(x) \mathrm{d} x\right]} & \text { for }(1) \text { - differentiability } \\ {\left[\int_{a}^{t} F_{2 r}^{\prime}(x) \mathrm{d} x, \int_{a}^{t} F_{1 r}^{\prime}(x) \mathrm{d} x\right]} & \text { for }(2) \text { - differentiability }\end{cases}
$$

This analysis deals with $\mathrm{ABC}$ gH-derivative but before presenting its definition, it is worth recalling the Caputo and $\mathrm{ABC}$ fractional derivatives of crisp functions. Hereunder, the underlying notations will be used. $L_{p}[a, b]=\left\{f:[a, b] \rightarrow \mathbb{R}, \int_{a}^{b}|f(x)|^{p} \mathrm{~d} x<\infty, 1 \leq p<\infty\right\} ; C^{F}[a, b]$ refers to the space of all continuous fuzzy 
valued functions on $[a, b] ; H^{1}(a, b)=\left\{F: F, F^{\prime} \in L_{2}(a, b)\right\}$ refers to usual Sobolev space; $L_{p}^{F}[a, b]=\{F$ : $[a, b] \rightarrow \mathbb{R}_{\mathcal{F}}, F$ is measurable and $\left.\int_{a}^{b} D(F(x), 0)^{p} \mathrm{~d} x<\infty, 1 \leq \mathrm{p}<\infty\right\}$, as well as $A \mathcal{C}[0, \infty)=\{f:[0, \infty) \rightarrow \mathbb{R}$ is absolutely continuous on $[0, \infty)\}$.

Definition 2.6. [42] The Caputo fractional derivative (CFD) operator of order $\beta>0$ for a function $g(x) \in$ $A \mathcal{C}[a, b]$ is given as

$$
{ }^{C} D_{a+}^{\beta} g(x)=\frac{1}{\Gamma(n-\beta)} \int_{a}^{x} \frac{g^{(n)}(\tau)}{(x-\tau)^{\beta-n+1}} \mathrm{~d} \tau
$$

where $n-1<\beta \leq n, n \in \mathbb{N}, x>a$. Specifically, we have ${ }^{C} D_{a+}^{\beta} g(x)=\frac{1}{\Gamma(1-\beta)} \int_{a}^{x} \frac{g^{\prime}(\tau)}{(x-\tau)^{\beta}} \mathrm{d} \tau$ for $0<\beta<1$.

The CFD possesses a kernel with singularity that includes the memory effect, so this definition cannot describe the full effect of memory. Hereby, we present the Atangana-Baleanu definition of Caputo-type in which the singular kernel will be replaced by the Mittag-Leffler function.

Definition 2.7. [21] The Atangana-Baleanu fractional derivative of Caputo type (ABC) of order $\beta \in[0,1]$ for a function $g(x) \in H^{1}(a, b)$ is given as

$$
{ }^{A B C} D_{a}^{\beta} g(x)=\frac{\kappa(\beta)}{1-\beta} \int_{a}^{x} g^{\prime}(\tau) E_{\beta}\left(-\beta \frac{(x-\tau)^{\beta}}{1-\beta}\right) \mathrm{d} \tau=\frac{\kappa(\beta)}{1-\beta} g^{\prime}(x) * E_{\beta}\left(\frac{-\beta x^{\beta}}{1-\beta}\right),
$$

provided that $\kappa(\beta)$ is a normalization constant so that $\kappa(0)=\kappa(1)=1$. Specifically, when $\kappa(\beta)=1$, the $\mathrm{ABC}$ derivative can be presented as follows

$$
{ }^{A B C} D_{a}^{\beta} g(x)=\frac{1}{1-\beta} \int_{a}^{x} g^{\prime}(\tau) E_{\beta}\left(-\beta \frac{(x-\tau)^{\beta}}{1-\beta}\right) \mathrm{d} \tau, 0<\beta \leq 1
$$

where the symbol $(*)$ denotes the convolution.

Definition 2.8. [21] The integral associated with the $\mathrm{ABC}$ derivative of order $\beta$ is given as:

$$
{ }_{a}^{A B} I^{\beta}(g(x))=\frac{1-\beta}{\kappa(\beta)} g(x)+\frac{\beta}{\kappa(\beta) \Gamma(\beta)} \int_{a}^{x} g(\tau)(x-\tau)^{\beta-1} \mathrm{~d} \tau, 0<\beta \leq 1 .
$$

For $\beta=1$, we obtain the classical integral as well as for $\beta=0$, the $\mathrm{ABC}$ integral becomes the identity operator.

Note that the $\mathrm{ABC}$ derivative of any constant is zero, i.e., ${ }^{A B C} D_{a}^{\beta} \mathrm{C}=0$ for any constant $C$. Further, the composition relation between the $\mathrm{ABC}$ derivative and integral [23] so that ${ }_{a}^{A B} I^{\beta}\left({ }^{A B C} D_{a}^{\beta} g(x)\right)=g(x)-g(a)$. Furthermore, in order to have an average equals to 1 in Definition 2.8, put $\frac{1-\beta}{\kappa(\beta)}+\frac{\beta}{\kappa(\beta) \Gamma(\beta)}=1$ leads to $\kappa(\beta)=$ $1-\beta+\frac{\beta}{\Gamma(\beta)}$. The generalized H-differentiability was used to expand the definitions of fractional derivatives in the crisp sense for the fuzzy space as follows.

Definition 2.9. [48] Let $0<\beta<1, F:[\mathrm{a}, \mathrm{b}] \rightarrow \mathbb{R}_{\mathcal{F}}$ and $F \in C^{F}[a, b] \cap L^{F}[a, b]$. Then, $F$ is said to be Caputo's gH-differentiable at $x$ if $\left({ }^{\mathrm{C}} D_{a+}^{\beta} F\right)(x)=\frac{1}{\Gamma(1-\beta)} \int_{a}^{x} \frac{F^{\prime}(t)}{(x-t)^{\beta}} \mathrm{d} t$ exists. We say that $F$ is ${ }^{c}[(1)-\beta]$-differentiable if $F$ is (1)-differentiable, and $F$ is ${ }^{c}[(2)-\beta]$-differentiable if $F$ is (2)-differentiable.

Theorem 2.10. [48] Let $0<\beta<1, F:[a, b] \rightarrow \mathbb{R}_{\mathcal{F}}$ and $F \in A C^{F}[a, b]$ with $[F(x)]^{r}=\left[F_{1 r}(x), F_{2 r}(x)\right]$. Then, the fuzzy CFD exists almost everywhere on $(a, b)$ so that 
$-\left[\left({ }_{1-g H}^{C} D_{a+}^{\beta} F\right)(x)\right]^{r}=\left[\left({ }^{C} D_{a+}^{\beta} F_{1 r}\right)(x),\left({ }^{C} D_{a+}^{\beta} F_{2 r}\right)(x)\right]$, if $F$ is $\underset{g H}{C}[(1)-\beta]$-differentiable,
$\left.-\left[{ }_{2-g H}{ }_{2-g+}^{C} D_{a+}^{\beta} F\right)(x)\right]^{r}=\left[\left({ }^{C} D_{a+}^{\beta} F_{2 r}\right)(x),\left({ }^{C} D_{a+}^{\beta} F_{1 r}\right)(x)\right]$, if $F$ is $\underset{g H}{C}[(2)-\beta]$-differentiable.

Definition 2.11. [4] Let $0<\beta<1, \mathrm{~F}:[\mathrm{a}, \mathrm{b}] \rightarrow \mathbb{R}_{\mathcal{F}}$ and $F \in C^{F}[a, b] \cap L^{F}[a, b]$ with $[F(x)]^{r}=\left[F_{1 r}(x), F_{2 r}(x)\right]$. Then, the fuzzy gH-Atangana-Baleanu-Caputo fractional differentiable fuzzy-valued function (ABC $\mathrm{gH}-$ differentiability) is defined by means of the underlying two cases:

- If $\underset{g H}{A B C}[(1)-\beta]$-differentiability: $\left.{ }_{1-g H}^{A B C} D_{a+}^{\beta} F\right)(x)=\frac{\kappa(\beta)}{1-\beta} \int_{a}^{x} 1-g H D F(\tau) E_{\beta}\left(-\beta \frac{(x-\tau)^{\beta}}{1-\beta}\right) \mathrm{d} \tau$,

- If $\underset{g H}{A B C}[(2)-\beta]$-differentiability: $\left({ }_{2-g H}^{A B C} D_{a+}^{\beta} F\right)(x)=\frac{\kappa(\beta)}{1-\beta} \int_{a}^{x}{ }_{2-g H} D F(\tau) \quad E_{\beta}\left(-\beta \frac{(x-\tau)^{\beta}}{1-\beta}\right) \mathrm{d} \tau$.

Consequently, the interval parametric forms in the two cases can be deduced from Lebesgue integral in (2.2) as follows:

$$
\begin{aligned}
& -\left[\left({ }_{1-g H}^{A B C} D_{a+}^{\beta} F\right)(x)\right]^{r}=\left[\left({ }_{1}^{A B C} D_{a+}^{\beta} F_{1 r}\right)(x),\left(\underset{1}{A B C} D_{a+}^{\beta} F_{2 r}\right)(x)\right], \\
& -\left[\left({ }_{2-g H}^{A B C} D_{a+}^{\beta} F\right)(x)\right]^{r}=\left[\left({ }_{2}^{A B C} D_{a+}^{\beta} F_{2 r}\right)(x),\left(\underset{2}{A B C} D_{a+}^{\beta} F_{1 r}\right)(x)\right],
\end{aligned}
$$

where

$$
\begin{aligned}
& \left({ }_{i}^{A B C} D_{a+}^{\beta} F_{1 r}\right)(x)=\left.\frac{\kappa(\beta)}{1-\beta} \int_{a}^{x} F_{1 r}^{\prime}(\tau) E_{\beta}\left(-\beta \frac{(x-\tau)^{\beta}}{1-\beta}\right)\right|_{E_{\beta} \geq 0} \mathrm{~d} \tau+\left.\frac{\kappa(\beta)}{1-\beta} \int_{a}^{x} F_{2 r}^{\prime}(\tau) E_{\beta}\left(-\beta \frac{(x-\tau)^{\beta}}{1-\beta}\right)\right|_{E_{\beta}<0} \mathrm{~d} \tau, \\
& \left({ }_{i}^{A B C} D_{a+}^{\beta} F_{2 r}\right)(x)=\left.\frac{\kappa(\beta)}{1-\beta} \int_{a}^{x} F_{2 r}^{\prime}(\tau) E_{\beta}\left(-\beta \frac{(x-\tau)^{\beta}}{1-\beta}\right)\right|_{E_{\beta} \geq 0} \mathrm{~d} \tau+\left.\frac{\kappa(\beta)}{1-\beta} \int_{a}^{x} F_{1 r}^{\prime}(\tau) E_{\beta}\left(-\beta \frac{(x-\tau)^{\beta}}{1-\beta}\right)\right|_{E_{\beta}<0} \mathrm{~d} \tau,
\end{aligned}
$$

for $i=1,2$. Moreover, the integral associated with the $\mathrm{ABC}$ gH-derivative can be formulated in interval representation as

$$
\begin{aligned}
{\left[\left({ }^{A B C} I_{a+}^{\beta} F\right)(x)\right]^{r}=} & {\left[\left({ }^{A B C} I_{a+}^{\beta} F_{1 r}\right)(x),\left({ }^{A B C} I_{a+}^{\beta} F_{2 r}\right)(x)\right] } \\
= & {\left[\frac{1-\beta}{\kappa(\beta)} F_{1 r}(x)+\frac{\beta}{\kappa(\beta) \Gamma(\beta)} \int_{a}^{x} F_{1 r}(\tau)(x-\tau)^{\beta-1} \mathrm{~d} \tau, \frac{1-\beta}{\kappa(\beta)} F_{2 r}(x)\right.} \\
& \left.+\frac{\beta}{\kappa(\beta) \Gamma(\beta)} \int_{a}^{x} F_{2 r}(\tau)(x-\tau)^{\beta-1} \mathrm{~d} \tau\right]
\end{aligned}
$$

It is worth to be mentioned that it was shown in [4] that for $0 \leq r \leq 1$,

$$
{ }^{A B C} I_{a+}^{\beta}\left({ }^{A B C} D_{a+}^{\beta} F\right)(x)=\left.(F(x)-F(0))\right|_{E_{\beta} \geq 0}-\left.(-1)(F(x)-F(0))\right|_{E_{\beta}<0} .
$$

\section{ABC gH-DifFERENTIABILITY FORMULATION OF FFIDEs}

In this section, we adopt the interval ABC gH-derivative to handle a general form of Fredholm-Volterra FFIDE (1.1). Without loss of generality, we assume that the kernels are separable such that $H(t, \tau, x(\tau))=h(t, \tau) x(\tau)$ for $\tau \in[a, b]$ and $K(t, \tau, x(\tau))=k(t, \tau) x(\tau)$ for $\tau \in[a, t]$ with $h(t, \tau) \geq 0$ for $\tau \in\left[a, c_{1}\right], h(t, \tau) \leq 0$ for $\tau \in\left[c_{1}, b\right], \quad k(t, \tau) \geq$ 0 for $\tau \in\left[a, c_{2}\right]$ and $k(t, \tau) \leq 0$ for $\tau \in\left[c_{2}, t\right]$. Moreover, we suppose that $p(t) \geq 0$ on $[a, b]$.

To perform the procedure, write $f(t)$ and $\omega(t)$ in term of their $r$-cut representations: $[f(t)]^{r}=\left[f_{1 r}(t), f_{2 r}(t)\right]$, $[\omega(t)]^{r}=\left[\omega_{1 r}(t), \omega_{2 r}(t)\right]$ and $[\omega(a)]^{r}=\left[\omega_{1 r}(a), \omega_{2 r}(a)\right]=\left[\alpha_{1 r}, \alpha_{2 r}\right]$. Hence, the interval parametric form of FFIDE 
(1.1) is

$$
\left.\left.\left[\left({ }_{g H}^{A B C} D_{a^{+}}^{\beta} \omega\right)(t)\right]^{r}=p(t)[\omega(t)]^{r}+\int_{a}^{b}[h(t, \tau) \omega(\tau))\right]^{r} \mathrm{~d} \tau+\int_{a}^{t}[k(t, \tau) \omega(\tau))\right]^{r} \mathrm{~d} \tau+[f(t)]^{r},[\omega(a)]^{r}=[\alpha]^{r}
$$

Thus, the FFIDE (1.1) can be translated into one of the underlying systems:

- In the case of $\underset{g H}{A B C}[(1)-\beta]$-differentiability, we have

$$
\begin{aligned}
& \frac{\kappa(\beta)}{1-\beta} \int_{a}^{x}\left(\left.\omega_{1 r}^{\prime}(\tau) E_{\beta}\left(-\beta \frac{(x-\tau)^{\beta}}{1-\beta}\right)\right|_{E_{\beta} \geq 0}+\left.\omega_{2 r}^{\prime}(\tau) E_{\beta}\left(-\beta \frac{(x-\tau)^{\beta}}{1-\beta}\right)\right|_{E_{\beta}<0}\right) \mathrm{d} \tau \\
& =p(t) \omega_{1 r}(t)+\int_{a}^{c_{1}} h(t, \tau) \omega_{1 r}(t) \mathrm{d} \tau+\int_{c_{1}}^{b} h(t, \tau) \omega_{2 r}(t) \mathrm{d} \tau \\
& +\int_{a}^{c_{2}} k(t, \tau) \omega_{1 r}(t) \mathrm{d} \tau+\int_{c_{2}}^{t} k(t, \tau) \omega_{2 r}(t) \mathrm{d} \tau+f_{1 r}(t), \\
& \frac{\kappa(\beta)}{1-\beta} \int_{a}^{x}\left(\left.\omega_{2 r}^{\prime}(\tau) E_{\beta}\left(-\beta \frac{(x-\tau)^{\beta}}{1-\beta}\right)\right|_{E_{\beta} \geq 0}+\left.\omega_{1 r}^{\prime}(\tau) E_{\beta}\left(-\beta \frac{(x-\tau)^{\beta}}{1-\beta}\right)\right|_{E_{\beta}<0}\right) \mathrm{d} \tau \\
& =p(t) \omega_{2 r}(t)+\int_{a}^{c_{1}} h(t, \tau) \omega_{2 r}(t) \mathrm{d} \tau+\int_{c_{1}}^{b} h(t, \tau) \omega_{1 r}(t) \mathrm{d} \tau \\
& +\int_{a}^{c_{2}} k(t, \tau) \omega_{2 r}(t) \mathrm{d} \tau+\int_{c_{2}}^{t} k(t, \tau) \omega_{1 r}(t) \mathrm{d} \tau+f_{2 r}(t)
\end{aligned}
$$

- In the case of $\underset{g H}{A B C}[(2)-\beta]$-differentiability, we have

$$
\begin{aligned}
& \frac{\kappa(\beta)}{1-\beta} \int_{a}^{x}\left(\left.\omega_{1 r}^{\prime}(\tau) E_{\beta}\left(-\beta \frac{(x-\tau)^{\beta}}{1-\beta}\right)\right|_{E_{\beta} \geq 0}+\left.\omega_{2 r}^{\prime}(\tau) E_{\beta}\left(-\beta \frac{(x-\tau)^{\beta}}{1-\beta}\right)\right|_{E_{\beta}<0}\right) \mathrm{d} \tau \\
& =p(t) \omega_{2 r}(t)+\int_{a}^{c_{1}} h(t, \tau) \omega_{2 r}(t) \mathrm{d} \tau+\int_{c_{1}}^{b} h(t, \tau) \omega_{1 r}(t) \mathrm{d} \tau \\
& \quad+\int_{a}^{c_{2}} k(t, \tau) \omega_{2 r}(t) \mathrm{d} \tau+\int_{c_{2}}^{t} k(t, \tau) \omega_{1 r}(t) \mathrm{d} \tau+f_{2 r}(t), \\
& \frac{\kappa(\beta)}{1-\beta} \int_{a}^{x}\left(\left.\omega_{2 r}^{\prime}(\tau) E_{\beta}\left(-\beta \frac{(x-\tau)^{\beta}}{1-\beta}\right)\right|_{E_{\beta} \geq 0}+\left.\omega_{1 r}^{\prime}(\tau) E_{\beta}\left(-\beta \frac{(x-\tau)^{\beta}}{1-\beta}\right)\right|_{E_{\beta}<0}\right) \mathrm{d} \tau \\
& =p(t) \omega_{1 r}(t)+\int_{a}^{c_{1}} h(t, \tau) \omega_{1 r}(t) \mathrm{d} \tau+\int_{c_{1}}^{b} h(t, \tau) \omega_{2 r}(t) \mathrm{d} \tau \\
& \quad+\int_{a}^{c_{2}} k(t, \tau) \omega_{1 r}(t) \mathrm{d} \tau+\int_{c_{2}}^{t} k(t, \tau) \omega_{2 r}(t) \mathrm{d} \tau+f_{1 r}(t) ;
\end{aligned}
$$

along with the following initial conditions

$$
\omega_{1 r}(a)=\alpha_{1 r} \text { and } \omega_{2 r}(a)=\alpha_{2 r} .
$$


Algorithm 3.1. Let $\omega(t)$ be the unique analytical solution of the FFIDE (1.1). To obtain an interval approach for the approximate fuzzy solution of FFIDE (1.1) in the sense of ABC gH-differentiability using RKHS technique, perform the underlying steps:

Step 1: Take the $r$-cut for both sides of equation (1.1) in light of assuming $\underset{g H}{A B C}[(i)-\beta]$-differentiability, $i=1,2$.

Step 2: Convert equation (1.1) into the equivalent system of parametric ABC fractional IDEs (3.1) or (3.2) equipped with conditions (3.3).

Step 3: Use RKHS technique to approximate the parametric solutions for system (3.1) or (3.2) along with (3.3).

\section{ABC FFIDES IN TERMS OF RKHS ALGORITHM}

In this section, we present a quick review to some basic definitions and theorems concerning the reproducing kernel Theory. For this purpose, we recall the definition of direct sum of Hilbert spaces $W_{2}^{i}[a, b] \oplus W_{2}^{i}[a, b] ; i=1,2$. Subsequently, RKHS algorithm will be implemented to solve the ABC fuzzy fractional IDEs (3.1) or (3.2) along with (3.3). For more details, theorems, and applications for the reproducing kernel technique, the reader may refer to [18, 26, 35].

Definition 4.1. [18] Let $\mathcal{H}$ be Hilbert space over the set of complex numbers $\mathbb{C}$. A function $\mathcal{K}: \mathcal{M} \times \mathcal{M} \rightarrow \mathbb{C}$ is called a reproducing kernel of the Hilbert space $\mathcal{H}$ if and only if $\forall m \in \mathcal{M}$ and $\forall \mathfrak{h} \in \mathcal{H}$, we have $\mathcal{K}(\cdot,) \in \mathcal{H}$ and $(\mathfrak{h}(\cdot), \mathcal{K}(\cdot, m))=\mathfrak{h}(m)$.

The property of reproducing any element of $\mathcal{H}$ by the function $\mathcal{K}$ is called a reproducing property, in which the space $\mathcal{H}$ of this case is called a reproducing Kernel Hilbert space (RKHS). Some of the desirable complete RKHSs [26] are constructed as follows.

$-W_{2}^{1}[a, b]=\left\{\mathfrak{p}:[a, b] \rightarrow \mathbb{R}: \mathfrak{p} \in A C[a, b], \mathfrak{p}^{\prime} \in L_{2}[a, b]\right\}$ embedded with $\langle\mathfrak{p}, \mathfrak{q}\rangle_{W_{2}^{1}}=\mathfrak{p}(a) \mathfrak{q}(a)+\int_{a}^{b} \mathfrak{p}^{\prime}(t) \mathfrak{q}^{\prime}(t) \mathrm{d} t$ and $\|\mathfrak{p}\|_{W_{2}^{1}}=\sqrt{\langle\mathfrak{p}(t), \mathfrak{p}(t)\rangle_{W_{2}^{1}}} ; \mathfrak{p}, \mathfrak{q} \in W_{2}^{1}[a, b]$. The reproducing function is $\mathrm{R}_{\mathrm{t}}(s)= \begin{cases}1-a+t, & s \leq t, \\ 1-a+s, & s>t .\end{cases}$

$-W_{2}^{2}[a, b]=\left\{\mathfrak{p}: \mathfrak{p}, \mathfrak{p}^{\prime} \in A C[a, b], \mathfrak{p}^{\prime \prime} \in L_{2}[a, b], \mathfrak{p}(a)=0\right\}$ embedded with $\langle\mathfrak{p}, \mathfrak{q}\rangle_{W_{2}^{2}}=\mathfrak{p}^{\prime}(a) \mathfrak{q}^{\prime}(a)+\int_{a}^{b} \mathfrak{p}^{\prime \prime}(t) \mathfrak{q}^{\prime \prime}(t) \mathrm{d} t$ and $\|\mathfrak{p}\|_{W_{2}^{2}}=\sqrt{\langle\mathfrak{p}(t), \mathfrak{p}(t)\rangle_{W_{2}^{2}}} ; \mathfrak{p}, \mathfrak{q} \in W_{2}^{2}[a, b]$. The reproducing function is

$G_{t}(s)= \begin{cases}\frac{1}{6}(s-a)\left(2 a^{2}-s^{2}+3 t(2+s)-a(6+3 t+s)\right), & s \leq t, \\ \frac{1}{6}(t-a)\left(2 a^{2}-t^{2}+3 s(2+t)-a(6+3 s+t)\right), & s>t .\end{cases}$

Remark 4.2. [18] The image of the interval $[a, b]$ under the mappings $\mathrm{R}:[\mathrm{a}, \mathrm{b}] \times[\mathrm{a}, \mathrm{b}] \rightarrow \mathbb{R}$ and $G:[\mathrm{a}, \mathrm{b}] \times[\mathrm{a}, \mathrm{b}] \rightarrow \mathbb{R}$ is $1+[0, b-a]$ and $\frac{1}{6}(b-a)^{2}[-b-5 a, 3(a+b)+6]$, respectively.

Furthermore, the direct sums $W_{2}^{i}[a, b] \oplus W_{2}^{i}[a, b], i=1,2$ of these RKHS's are formulated as

$$
\begin{aligned}
& -\Theta[a, b]=W_{2}^{1}[a, b] \oplus W_{2}^{1}[a, b]=\left\{\left[\mathfrak{p}_{1}(t), \mathfrak{p}_{2}(t)\right]^{T}: \mathfrak{p}_{1}, \mathfrak{p}_{2} \in W_{2}^{1}\right\} \text { equipped with }\langle\mathfrak{p}(t), \mathfrak{q}(t)\rangle_{\Theta}=\sum_{i=1}^{2}\left\langle\mathfrak{p}_{i}(t), \mathfrak{q}_{i}(t)\right\rangle_{W_{2}^{1}} \\
& \text { and }\|\mathfrak{p}\|_{\Theta}=\sqrt{\sum_{i=1}^{2}\left\|\mathfrak{p}_{i}\right\|_{W_{2}^{1}}^{2}} ; \mathfrak{p}(t)=\left[\mathfrak{p}_{1}(t), \mathfrak{p}_{2}(t)\right]^{T}, \mathfrak{q}=\left[\mathfrak{q}_{1}(t), \mathfrak{q}_{2}(t)\right]^{T} \in \Theta[a, b] . \\
& -\Upsilon[a, b]=W_{2}^{2}[a, b] \oplus W_{2}^{2}[a, b]=\left\{\left[\mathfrak{p}_{1}(t), \mathfrak{p}_{2}(t)\right]^{T}: \mathfrak{p}_{1}, \mathfrak{p}_{2} \in W_{2}^{2}\right\} \text { equipped with }\langle\mathfrak{p}(t), \mathfrak{q}(t)\rangle_{\Upsilon}=\sum_{i=1}^{2}\left\langle\mathfrak{p}_{i}(t), \mathfrak{q}_{i}(t)\right\rangle_{W_{2}^{2}} \\
& \quad \text { and }\|\mathfrak{p}\|_{\Upsilon}=\sqrt{\sum_{i=1}^{2}\left\|\mathfrak{p}_{i}\right\|_{W_{2}^{2}}^{2}} ; \mathfrak{p}(t)=\left[\mathfrak{p}_{1}(t), \mathfrak{p}_{2}(t)\right]^{T}, \mathfrak{q}=\left[\mathfrak{q}_{1}(t), \mathfrak{q}_{2}(t)\right]^{T} \in \Upsilon[a, b] .
\end{aligned}
$$

In the following, the RKHS method is implemented to obtain approximate and analytic solutions of FFIDE (1.1) in interval parametric form under assuming of $\underset{g H}{A B C}[(1)-\beta]$-differentiability. While the same procedures can be employed for $\underset{g H}{A B C}[(2)-\beta]$-differentiability. Towards this end, define the integrodifferential operators $l_{i j}: W_{2}^{2}[a, b] \rightarrow W_{2}^{1}[a, b], i, j=$ 1,2 , such that

$l_{i j} z(t)=\left\{\begin{array}{l}\left.\frac{\kappa(\beta)}{1-\beta} \int_{a}^{t} z^{\prime}(\tau) E_{\beta}\left(-\beta \frac{(t-\tau)^{\beta}}{1-\beta}\right)\right|_{E_{\beta} \geq 0} \mathrm{~d} \tau-p(t) z(t)-\int_{a}^{c_{1}} h(t, \tau) z(\tau) \mathrm{d} \tau-\int_{a}^{c_{2}} k(t, \tau) z(\tau) \mathrm{d} \tau, i=j, \\ \left.\frac{\kappa(\beta)}{1-\beta} \int_{a}^{t} z^{\prime}(\tau) E_{\beta}\left(-\beta \frac{(t-\tau)^{\beta}}{1-\beta}\right)\right|_{E_{\beta}<0} \mathrm{~d} \tau-\int_{c_{1}}^{b} h(t, \tau) z(\tau) \mathrm{d} \tau--\int_{c_{2}}^{t} k(t, \tau) z(\tau) \mathrm{d} \tau, \quad i \neq j .\end{array}\right.$ 
In this direction, put $F_{r}=\left[\begin{array}{l}f_{1 r} \\ f_{2 r}\end{array}\right], \omega_{r}=\left[\begin{array}{c}\omega_{1 r} \\ \omega_{2 r}\end{array}\right], \alpha_{r}=\left[\begin{array}{c}\alpha_{1 r} \\ \alpha_{2 r}\end{array}\right]$, and $L=\left[\begin{array}{ll}l_{11} & l_{12} \\ l_{21} & l_{22}\end{array}\right]$ so that $L: \Upsilon[a, b] \rightarrow$ $\Theta[a, b]$, in which the system (3.1) along with conditions (3.3) can be rewritten as $L \omega_{r}(t)=F_{r}(t), \omega_{r}(0)=\alpha_{r}$. With the aid of transform $\xi_{r}(t)=\left[\begin{array}{l}\xi_{1 r}(t) \\ \xi_{2 r}(t)\end{array}\right]=\omega_{r}(t)-\alpha_{r}$ to homogenize the conditions (3.1), it yields

$$
\left\{\begin{array}{l}
\left(L \xi_{r}\right)(t)=Q_{r}(t)=\left[\begin{array}{l}
q_{1 r}(t) \\
q_{2 r}(t)
\end{array}\right], \\
\xi_{r}(a)=0,
\end{array}\right.
$$

where

$$
\begin{aligned}
& q_{1 r}(t)=\alpha_{1 r}\left(p(t)+\int_{a}^{c_{1}} h(t, \tau) \mathrm{d} \tau+\int_{a}^{c_{2}} k(t, \tau) \mathrm{d} \tau\right)+\alpha_{2 r}\left(\int_{c_{1}}^{b} h(t, \tau)+\int_{c_{2}}^{t} k(t, \tau) \mathrm{d} \tau \mathrm{d} \tau\right)+f_{1 r}(t), \\
& q_{2 r}(t)=\alpha_{2 r}\left(p(t)+\int_{a}^{c_{1}} h(t, \tau) \mathrm{d} \tau+\int_{a}^{c_{2}} k(t, \tau) \mathrm{d} \tau\right)+\alpha_{1 r}\left(\int_{c_{1}}^{b} h(t, \tau)+\int_{c_{2}}^{t} k(t, \tau) \mathrm{d} \tau \mathrm{d} \tau\right)+f_{2 r}(t) .
\end{aligned}
$$

Theorem 4.3. The operator $L: \Upsilon[a, b] \rightarrow \Theta[a, b]$ is linear and bounded.

Proof. We firstly prove that the operators $l_{i j}: W_{2}^{2}[a, b] \rightarrow W_{2}^{1}[a, b], i, j=1,2$, are bounded and linear. The linearity is clear so for boundedness, $\forall \xi_{j r} \in W_{2}^{2}[a, b], j=1,2$, we have

$$
\left\|l_{i j} \xi_{j r}\right\|_{W_{2}^{1}}^{2}=\left\langle l_{i j} \xi_{j r}, l_{i j} \xi_{j r}\right\rangle_{W_{2}^{1}}=\left[\left(l_{i j} \xi_{j r}\right)(a)\right]^{2}+\int_{a}^{b}\left[\left(l_{i j} \xi_{j r}\right)^{\prime}(t)\right]^{2} \mathrm{~d} t .
$$

By the reproducing property of $G_{\mathrm{t}}(\mathrm{s})$, one can write $\xi_{j r}(t)=\left\langle\xi_{j r}(s), G_{t}(s)\right\rangle_{W_{2}^{2}}$ so that

$$
\begin{gathered}
\left(l_{i j} \xi_{j r}\right)(t)=\left\langle\xi_{j r}(s),\left(l_{i j} G_{t}(s)\right)\right\rangle_{W_{2}^{2}}, \\
\left(l_{i j} \xi_{j r}\right)^{\prime}(t)=\left\langle\xi_{j r}(s), \frac{\mathrm{d}}{\mathrm{d} t}\left(l_{i j} G_{t}(s)\right)\right\rangle_{W_{2}^{2}} .
\end{gathered}
$$

Using Schwarz Inequality and the continuity of $G_{t}(s)$ over the closed interval $[a, b]$, we get

$$
\begin{gathered}
\left|\left(l_{i j} \xi_{j r}\right)(t)\right|=\left|\left\langle\xi_{j r}(s),\left(l_{i j} G_{t}\right)(s)\right\rangle_{W_{2}^{2}}\right| \leq\left\|\xi_{j r}\right\|_{W_{2}^{2}}\left\|\left(l_{i j} G_{t}\right)(s)\right\|_{W_{2}^{1}} \leq M_{i j}\left\|\xi_{j r}\right\|_{W_{2}^{2}}, \\
\left|\left(l_{i j} \xi_{j r}\right)^{\prime}(t)\right|=\left|\left\langle\xi_{j r}(s), \frac{\mathrm{d}}{\mathrm{d} t}\left(l_{i j} G_{t}\right)(s)\right\rangle_{W_{2}^{2}}\right| \leq\left\|\xi_{j r}\right\|_{W_{2}^{2}}\left\|\frac{\mathrm{d}}{\mathrm{d} t}\left(l_{i j} G_{t}\right)(s)\right\|_{W_{2}^{1}} \leq N_{i j}\left\|\xi_{j r}\right\|_{W_{2}^{2}},
\end{gathered}
$$

where $M_{i j}, N_{i j} \in \mathbb{R}^{+}$.

Therefore, $\left\|l_{i j} \xi_{j r}\right\|_{W_{2}^{1}}^{2} \leq\left(M_{i j}^{2}+N_{i j}^{2}(b-a)\right)\left\|\xi_{j r}\right\|_{W_{2}^{2}}^{2}$, which leads to $\left\|l_{i j} \xi_{j r}\right\|_{W_{2}^{1}} \leq T_{i j}\left\|\xi_{j r}\right\|_{W_{2}^{2}}$, where $T_{i j}=$ $\sqrt{\left(M_{i j}^{2}+N_{i j}^{2}(b-a)\right)}$. Hence, $l_{i j}, i, j=1,2$, are bounded operators.

For the boundedness of $\mathrm{p}$, let $\xi_{r} \in \Upsilon[a, b]$, we have

$$
\begin{aligned}
\left\|l \xi_{r}\right\|_{\Theta} & =\left\|\left[\begin{array}{c}
\sum_{j=1}^{2} l_{1 j} \xi_{j r} \\
\sum_{j=1}^{2} l_{2 j} \xi_{j r}
\end{array}\right]\right\|_{\Theta}=\sqrt{\sum_{i=1}^{2}\left(\left\|\sum_{j=1}^{2} l_{i j} \xi_{j r}\right\|\right)^{2}} \leq \sqrt{\sum_{i=1}^{2}\left(\sum_{j=1}^{2}\left\|l_{i j}\right\|\left\|\xi_{j r}\right\|_{W_{2}^{2}}\right)^{2}} \\
& \leq \sqrt{\sum_{i=1}^{2}\left(\sum_{j=1}^{2}\left\|l_{i j}\right\|^{2}\right)\left(\sum_{j=1}^{2}\left\|\xi_{j r}\right\|_{W_{2}^{2}}^{2}\right)} \leq \sqrt{\sum_{i=1}^{2} \sum_{j=1}^{2}\left\|l_{i j}\right\|^{2}}\left\|\xi_{r}\right\|_{\Upsilon} .
\end{aligned}
$$


Therefore, since $l_{i j}, j=1,2$ is bounded, then $L$ is so.

To construct a complete function system on the space $\Upsilon[a, b]$ in the view of $l_{i j}, i, j=1,2$, pick a dense countable set $\left\{t_{k}\right\}_{k=1}^{\infty}$ in $[a, \mathrm{~b}]$ and let $l_{i j}^{*}$ be the adjoint operator of $l_{i j}$ so that $\left[l_{i j}^{*} R_{t_{k}}\right](t)=\left\langle\left[l_{i j}^{*} R_{t_{k}}\right](s), G_{t}(s)\right\rangle_{W_{2}^{2}}=$ $\left\langle R_{t_{k}}(s),\left[L_{i j} G_{t}(s)\right]\right\rangle_{W_{2}^{1}}=\left.l_{i j} G_{t}(s)\right|_{s=t_{k}}$. Meanwhile, we define $\Psi_{k j}(t)=\left[\left.l_{j 1} G_{t}(s)\right|_{s=t_{k}},\left.l_{j 2} G_{t}(s)\right|_{s=t_{k}}\right]^{T}, k=$ $1,2,3, \ldots, j=1,2$.

Theorem 4.4. Let $\left\{t_{k}\right\}_{k=1}^{\infty}$ be dense on $[a, b]$ and assume that the system (3.1) along with ICs (3.3) has a unique solution, then $\left\{\Psi_{k j}(t)\right\}_{(k, j)=(1,1)}^{(\infty, 2)}$ forms a complete function system of $\Upsilon[a, b]$.

Proof. First of all, we show that the set $\left\{\Psi_{k j}(t)\right\}_{(k, j)=(1,1)}^{(\infty, 2)}$ is linearly independent in $\Upsilon[a, b]$, then we prove the completeness of such function system. To achieve this, assume on the contrary that $\left\{\Psi_{k_{1} j_{1}}(t), \Psi_{k_{2} j_{2}}(t), \Psi_{k_{3} j_{3}}(t), \ldots, \Psi_{k_{m} j_{m}}(t)\right\}$ is a linearly dependent subset of $\left\{\Psi_{k j}(t)\right\}_{(k, j)=(1,1)}^{(\infty, 2)}$. Let $r=$ $\max \left\{k_{1}, k_{2}, k_{3}, \ldots, k_{m}\right\}$ and put $v_{k}(s)=R_{t_{k}}(s)$. So, $\left\{\Psi_{k j}(t)\right\}_{(k, j)=(1,1)}^{(\infty, 2)}$ is linearly dependent, i.e., there are scalars $c_{k j}, j=1,2, k=1,2, \ldots, r$, not all are zeros such that $\sum_{j=1}^{2} \sum_{k=1}^{r} c_{k j} \Psi_{k j}(t)=0$. For Y $=\left[\begin{array}{l}y_{1} \\ y_{2}\end{array}\right] \in \Upsilon[a, b]$, we have

$$
\begin{aligned}
& \left\langle L Y(t),\left[\begin{array}{l}
\sum_{k=1}^{r} c_{k 1} v_{k}(t) \\
\sum_{k=1}^{r} c_{k 2} v_{k}(t)
\end{array}\right]\right\rangle_{\Theta}=\left\langle\left[\begin{array}{ll}
l_{11} & l_{12} \\
l_{21} & l_{22}
\end{array}\right]\left[\begin{array}{l}
y_{1}(t) \\
y_{2}(t)
\end{array}\right],\left[\begin{array}{l}
\sum_{k=1}^{r} c_{k 1} v_{k}(t) \\
\sum_{k=1}^{r} c_{k 2} v_{k}(t)
\end{array}\right]_{\Theta}\right. \\
& =\left\langle\left[\begin{array}{l}
\sum_{j=1}^{2} l_{1 j} y_{j}(t) \\
\sum_{j=1}^{2} l_{2 j} y_{j}(t)
\end{array}\right],\left[\begin{array}{l}
\sum_{k=1}^{r} c_{k 1} v_{k}(t) \\
\sum_{k=1}^{r} c_{k 2} v_{k}(t)
\end{array}\right]\right\rangle_{\Theta} \\
& =\left\langle\sum_{j=1}^{2} l_{1 j} y_{j}(t), \sum_{k=1}^{r} c_{k 1} v_{k}(t)\right\rangle_{W_{2}^{1}}+\left\langle\sum_{j=1}^{2} l_{2 j} y_{j}(t), \sum_{k=1}^{r} c_{k 2} v_{k}(t)\right\rangle_{W_{2}^{1}} \\
& =\sum_{j=1}^{2} \sum_{k=1}^{r}\left\langle l_{1 j} y_{j}(t), c_{k 1} v_{k}(t)\right\rangle_{W_{2}^{1}}+\sum_{j=1}^{2} \sum_{k=1}^{r}\left\langle l_{2 j} y_{j}(t), c_{k 2} v_{k}(t)\right\rangle_{W_{2}^{1}} \\
& =\sum_{j=1}^{2} \sum_{k=1}^{r}\left\langle y_{j}(t), l_{1 j}^{*} c_{k 1} v_{k}(t)\right\rangle_{W_{2}^{2}}+\sum_{j=1}^{2} \sum_{k=1}^{r}\left\langle y_{j}(t), l_{2 j}^{*} c_{k 2} v_{k}(t)\right\rangle_{W_{2}^{2}} \\
& =\left\langle\left[\begin{array}{c}
\sum_{j=1}^{2} y_{j}(t) \\
\sum_{j=1}^{2} y_{j}(t)
\end{array}\right],\left[\begin{array}{l}
\sum_{k=1}^{r} l_{1 j}^{*} c_{k 1} v_{k}(t) \\
\sum_{k=1}^{r} l_{2 j}^{*} c_{k 2} v_{k}(t)
\end{array}\right]\right\rangle_{\Upsilon} \\
& =\left\langle\left[\begin{array}{l}
y_{1}(t) \\
y_{2}(t)
\end{array}\right],\left[\begin{array}{l}
\sum_{j=1}^{2} \sum_{k=1}^{r} l_{1 j}^{*} c_{k 1} v_{k}(t) \\
\sum_{j=1}^{2} \sum_{k=1}^{r} l_{2 j}^{*} c_{k 2} v_{k}(t)
\end{array}\right]\right\rangle_{\Upsilon} \\
& =\left\langle\mathrm{Y}(t), \sum_{j=1}^{2} \sum_{k=1}^{r} c_{k j} \Psi_{k j}(t)\right\rangle_{\Upsilon}=0 \text {. }
\end{aligned}
$$

Consequently, $\left\langle L \mathrm{Y}(t),\left[\begin{array}{c}\sum_{k=1}^{r} c_{k 1} v_{k}(t) \\ \sum_{k=1}^{r} c_{k 2} v_{k}(t)\end{array}\right]\right\rangle_{\Theta}=0$. Due to Theorem 4.3, that is, $L$ is bounded, and by the uniqueness of the solution, it yields $\left[\begin{array}{l}\sum_{k=1}^{r} c_{k 1} v_{k}(t) \\ \sum_{k=1}^{r} c_{k 2} v_{k}(t)\end{array}\right]=0$.

Now, define $w_{k}(t) \in W_{2}^{1}[a, b]$ by $w_{r}(t)=\left\{\begin{array}{lr}1, & t=t_{r}, \\ 0, \quad t=t_{k}, k=1,2, \ldots, r, k \neq r & \text { otherwise. }\end{array}\right.$

Thus, $c_{i j}=\left\langle w_{i}(t), \sum_{k=1}^{r} c_{k j} v_{i}(t)\right\rangle_{W_{2}^{1}}=0, j=1,2, i=1,2, \ldots, r$, which contradicts the fact that not all $c_{i j}$ are zeros. Therefore, $\left\{\Psi_{k j}(t)\right\}_{(k, j)=(1,1)}^{(\infty, 2)}$ is linearly independent. 
To complete the proof, let $\mathrm{Y}(t)=\left[\begin{array}{l}y_{1}(t) \\ y_{2}(t)\end{array}\right] \in \Upsilon[a, b]$ with $\left\langle\mathrm{Y}(t), \Psi_{k j}(t)\right\rangle_{\Upsilon}=0$ for each $k=1,2,3, \ldots, j=1,2$, and use the linearly independence of $\left\{\Psi_{k j}(t)\right\}_{(k, j)=(1,1)}^{(\infty, 2)}$ to get

$$
\begin{aligned}
0=\left\langle\mathrm{Y}(t), \Psi_{k j}(t)\right\rangle_{\Upsilon} & =\left\langle\left[\begin{array}{l}
y_{1}(t) \\
y_{2}(t)
\end{array}\right],\left[\begin{array}{c}
\left.l_{j 1} G_{t}(s)\right|_{s=t_{k}} \\
\left.l_{j 2} G_{t}(s)\right|_{s=t_{k}}
\end{array}\right]\right\rangle_{\Upsilon} \\
& =\left\langle y_{1}(t),\left.l_{j 1} G_{t}(s)\right|_{s=t_{k}}\right\rangle_{W_{2}^{2}}+\left\langle y_{2}(t),\left.l_{j 2} G_{t}(s)\right|_{s=t_{k}}\right\rangle_{W_{2}^{2}} \\
& =\left\langle y_{1}(t),\left[\begin{array}{ll}
l_{j 1}^{*} & R_{t_{k}}
\end{array}\right](t)\right\rangle_{W_{2}^{2}}+\left\langle y_{2}(t),\left[l_{j 2}^{*} R_{t_{k}}\right](t)\right\rangle_{W_{2}^{2}} \\
& =l_{j 1} y_{1}\left(t_{k}\right)+l_{j 2} y_{2}\left(t_{k}\right) .
\end{aligned}
$$

Since $\left\{t_{k}\right\}_{k=1}^{\infty}$ is dense on $[a, b]$, we deduce that $l_{11} y_{1}(t)+l_{12} y_{2}(t)=0$ and $l_{21} y_{1}(t)+l_{22} y_{2}(t)=0$. Equivalently, $\left[\begin{array}{ll}l_{11} & l_{12} \\ l_{21} & l_{22}\end{array}\right]\left[\begin{array}{l}y_{1} \\ y_{2}\end{array}\right]=0$. Again, from the uniqueness of the solution, it follows $\mathrm{Y}(t)=0$. Hence, $\left\{\Psi_{k j}(t)\right\}_{(k, j)=(1,1)}^{(\infty, 2)}$ is the complete function system of $\Upsilon[a, b]$.

As a next step, we obtain the orthonormal function system for $\Upsilon[a, b]$, namely $\left\{\bar{\Psi}_{k j}(t)\right\}_{(k, j)=(1,1)}^{(\infty, 2)}$, of $\left\{\Psi_{k j}(t)\right\}_{(k, j)=(1,1)}^{(\infty, 2)}$ such that $\bar{\Psi}_{k j}(t)=\sum_{m=1}^{k} \lambda_{k j l} \Psi_{m j}(t), k=1,2,3, \ldots, j=1,2$. Here, $\lambda_{k j m}$ are the Gram-Schmidt orthogonalization coefficients. Afterwards, we use Fourier expansion to express the solution of equation (4.2) in a series form as in the underlying theorem.

Theorem 4.5. Let $\left\{t_{k}\right\}_{k=1}^{\infty}$ be dense on $[a, b]$ and $\xi_{r}(t)=\left[\begin{array}{l}\xi_{1 r}(t) \\ \xi_{2 r}(t)\end{array}\right] \in \Theta[\mathrm{a}, \mathrm{b}]$ be the unique solution of equation (4.2). Then, $\xi_{r}(t)$ has the analytic series representation:

$$
\xi_{r}(t)=\sum_{j=1}^{2} \sum_{k=1}^{\infty} \sum_{m=1}^{k} \lambda_{k j m} q_{j r}\left(t_{m}\right) \bar{\Psi}_{k j}(t)
$$

Proof. Assume that $\left\{t_{k}\right\}_{k=1}^{\infty}$ is dense on $[a, b]$ and expand the solution $\xi_{r}(t)$ in Fourier series about the complete function system $\left\{\bar{\Psi}_{k j}(t)\right\}_{(k, j)=(1,1)}^{(\infty, 2)}$ of $\Theta[a, b]$ as follows

$$
\begin{aligned}
& \xi_{r}(t)=\sum_{j=1}^{2} \sum_{k=1}^{\infty}\left\langle\xi_{r}(t), \bar{\Psi}_{k j}(t)\right\rangle_{\Upsilon} \bar{\Psi}_{k j}(t) \\
& =\sum_{j=1}^{2} \sum_{k=1}^{\infty}\left\langle\xi_{r}(t), \sum_{m=1}^{k} \lambda_{k j m} \Psi_{m j}(t)\right\rangle_{\Upsilon} \bar{\Psi}_{k j}(t) \\
& =\sum_{j=1}^{2} \sum_{k=1}^{\infty} \sum_{m=1}^{k} \lambda_{k j m}\left\langle\xi_{r}(t), \Psi_{m j}(t)\right\rangle_{\Upsilon} \bar{\Psi}_{k j}(t) \\
& =\sum_{j=1}^{2} \sum_{k=1}^{\infty} \sum_{m=1}^{k} \lambda_{k j m}\left\langle\left[\begin{array}{l}
\xi_{1 r}(t) \\
\xi_{2 r}(t)
\end{array}\right],\left[\begin{array}{l}
\left.l_{j 1} G_{t}(s)\right|_{s=t_{m}} \\
\left.l_{j 2} G_{t}(s)\right|_{s=t_{m}}
\end{array}\right]\right\rangle_{\Upsilon} \bar{\Psi}_{k j}(t) \\
& =\sum_{j=1}^{2} \sum_{k=1}^{\infty} \sum_{m=1}^{k} \lambda_{k j m}\left(\left\langle\xi_{1 r}(t),\left.l_{j 1} G_{t}(s)\right|_{s=t_{m}}\right\rangle_{W_{2}^{2}}+\left\langle\xi_{2 r}(t),\left.l_{j 2} G_{t}(s)\right|_{s=t_{m}}\right\rangle_{W_{2}^{2}}\right) \bar{\Psi}_{k j}(t) \\
& =\sum_{j=1}^{2} \sum_{k=1}^{\infty} \sum_{m=1}^{k} \lambda_{k j m}\left(\left\langle\xi_{1 r}(t), l_{j 1}^{*} R_{t_{m}}(t)\right\rangle_{W_{2}^{2}}+\left\langle\xi_{2 r}(t), l_{j 2}^{*} R_{t_{m}}(t)\right\rangle_{W_{2}^{2}}\right) \bar{\Psi}_{k j}(t) \\
& =\sum_{j=1}^{2} \sum_{k=1}^{\infty} \sum_{m=1}^{k} \lambda_{k j m}\left(\left\langle l_{j 1} \xi_{1 r}(t), R_{t_{m}}(t)\right\rangle_{W_{2}^{1}}+\left\langle l_{j 2} \xi_{2 r}(t), R_{t_{m}}(t)\right\rangle_{W_{2}^{1}}\right) \bar{\Psi}_{k j}(t) \\
& =\sum_{j=1}^{2} \sum_{k=1}^{\infty} \sum_{m=1}^{k} \lambda_{k j m}\left(l_{j 1} \xi_{1 r}\left(t_{m}\right)+l_{j 2} \xi_{2 r}\left(t_{m}\right)\right) \bar{\Psi}_{k j}(t)
\end{aligned}
$$




$$
\begin{aligned}
& =\sum_{k=1}^{\infty} \sum_{m=1}^{k} \lambda_{k 1 m}\left(l_{11} \xi_{1 r}\left(t_{m}\right)+l_{12} \xi_{2 r}\left(t_{m}\right)\right) \bar{\Psi}_{k 1}(t)+\sum_{k=1}^{\infty} \sum_{m=1}^{k} \lambda_{k 2 m}\left(l_{21} \xi_{1 r}\left(t_{m}\right)+l_{22} \xi_{2 r}\left(t_{m}\right)\right) \bar{\Psi}_{k 2}(t) \\
& =\sum_{k=1}^{\infty} \sum_{m=1}^{k} \lambda_{k 1 m} q_{1 r}\left(t_{m}\right) \bar{\Psi}_{k 1}(t)+\sum_{k=1}^{\infty} \sum_{m=1}^{k} \lambda_{k 2 m} q_{2 r}\left(t_{m}\right) \bar{\Psi}_{k 2}(t) \\
& =\sum_{j=1}^{2} \sum_{k=1}^{\infty} \sum_{m=1}^{k} \lambda_{k j m} q_{j r}\left(t_{m}\right) \bar{\Psi}_{k j}(t) .
\end{aligned}
$$

The Nth-term approximate solution of equation (4.2) can be obtained by truncating the finite sum $\xi_{r}^{N}(t)$ of the series solution (4.3) as follows.

$$
\xi_{r}^{N}(t)=\sum_{j=1}^{2} \sum_{k=1}^{\mathrm{N}} \sum_{m=1}^{k} \lambda_{k j m} q_{j r}\left(t_{m}\right) \bar{\Psi}_{k j}(t) .
$$

Theorem 4.6. The approximate solution $\xi_{r}^{N}(t)$ given in (4.4) converges uniformly to the analytic solution $\xi_{r}(t)$ presented in (4.3).

Proof. By using the reproducing property, Schwarz inequality together with the fact that the reproducing kernel $Д_{t}(s)$ of the space $W_{2}^{2}[a, b]$ is bounded over $[a, b]$, we have

$$
\begin{aligned}
\left|\xi_{r}(t)-\xi_{r}^{N}(t)\right| & =\left|\left[\begin{array}{l}
\xi_{1 r}(t) \\
\xi_{2 r}(t)
\end{array}\right]-\left[\begin{array}{l}
\xi_{1 r}^{N}(t) \\
\xi_{2 r}^{N}(t)
\end{array}\right]\right|=\left|\left\langle\left[\begin{array}{l}
\xi_{1 r}(s) \\
\xi_{2 r}(s)
\end{array}\right]-\left[\begin{array}{l}
\xi_{1 r}^{N}(s) \\
\xi_{2 r}^{N}(s)
\end{array}\right],\left[\begin{array}{l}
G_{t}(s) \\
G_{t}(s)
\end{array}\right]\right\rangle_{\Upsilon}\right| \\
& \leq\left\|\left[\begin{array}{l}
\xi_{1 r} \\
\xi_{2 r}
\end{array}\right]-\left[\begin{array}{l}
\xi_{1 r}^{N} \\
\xi_{2 r}^{N}
\end{array}\right]\right\|_{\Upsilon}\left\|\begin{array}{l}
G_{t}(s) \\
G_{t}(s)
\end{array}\right\|_{\Upsilon}=\left\|\left[\begin{array}{l}
\xi_{1 r}-\xi_{1 r}^{N} \\
\xi_{2 r}-\xi_{2 r}^{N}
\end{array}\right]\right\|_{\Upsilon} \sqrt{2\left(\left\|G_{t}(s)\right\|_{\Upsilon}\right)^{2}} \\
& =\sqrt{2}\left\|\xi_{r}-\xi_{r}^{N}\right\|_{\Upsilon}\left\|G_{t}(s)\right\|_{W_{2}^{2}} \leq \rho\left\|\xi_{r}(t)-\xi_{r}^{N}(t)\right\|_{\Upsilon} .
\end{aligned}
$$

From Remark 2.1, it yields $\rho=\sqrt{2}(b-a) \sqrt{\frac{1}{2}(a+b+2)}$. Meanwhile, $\left\|\xi_{r}-\xi_{r}^{N}\right\|_{\Upsilon} \rightarrow 0$ as $N \rightarrow \infty$. Therefore, $\xi_{r}^{N}(t)$ converges uniformly to $\xi_{r}(t)$.

\section{Numeric investigation of FFidEs With ABC gH-DIFFERENTIABILITY}

In this section, numerical solutions of various types FFIDEs with ABC gH-fractional derivative are investigated using RKHS algorithm. In specific, Volterra, Fredholm, and mixed Volterra-Fredholm FFIDEs are considered by taking different types of kernel functions, non-homogeneous terms, and fractional derivatives. The accuracy of our method is examined by comparing the exact, if exist, with RKHS approximate solutions. To see the effect of ABC gH-derivative on the behavior of the given FFIDEs, a comparison is also done between fuzzy approximate solutions under ABC gHdifferentiability with those of classical Caputo g-H-differentiability. In each example, the normalization coefficient is considered as $\kappa(\beta)=1-\beta+\frac{\beta}{\Gamma(\beta)}$. Our numerical results are carried out using Mathematica 10.

Example 5.1. Consider $H(t, \tau, \omega(\tau))=0, K(t, \tau, \omega(\tau))=\frac{1}{\Gamma(\beta)}(t-\tau)^{\beta-1} \omega(\tau), p(t)=0$, and $f(t)=\eta\left(1+t^{\beta}\right)$, where $\eta$ is the generalized triangular fuzzy number $(-1,0,1 ; 1)$, along with the fuzzy initial condition $\omega(0)=0$. Under such circumstance, FFIDE (1.1) converts into the Volterra FFIDE as:

$$
\left({ }_{g H}^{A B C} D_{0^{+}}^{\beta} \omega\right)(t)=\eta\left(1+t^{\beta}\right)+\frac{1}{\Gamma(\beta)} \int_{0}^{t}(t-\tau)^{\beta-1} \omega(\tau) \mathrm{d} \tau, \omega(0)=0,0<\beta \leq 1 .
$$

- For $\underset{g H}{A B C}[(1)-\beta]$-differentiability, the Volterra FFIDE (5.1) will be equivalent to the underlying crisp system of Volterra FIDEs: 

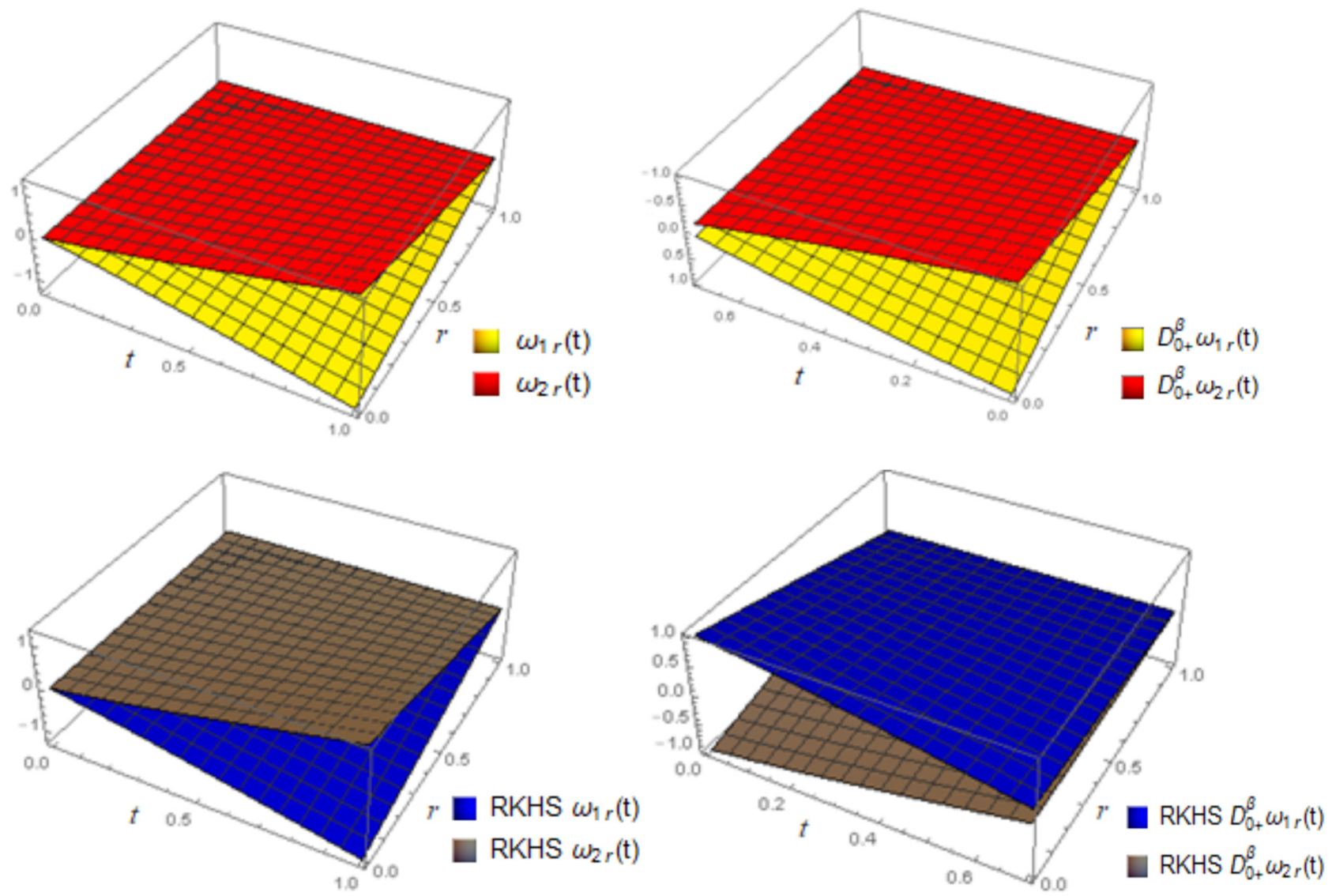

FIgURE 1. Comparison between the exact solution and its derivative (above) and approximate RKHS-solution (below) for $\beta=1$ under $\underset{g H}{A B C}[(1)-\beta]$-differentiability of Example 5.1, case 1.

$$
\begin{aligned}
& \left({ }_{1}^{A B C} D_{0^{+}}^{\beta} \omega_{1 r}\right)(t)=(r-1)\left(1+t^{\beta}\right)+\frac{1}{\Gamma(\beta)} \int_{0}^{t}(t-\tau)^{\beta-1} \omega_{1 r}(\tau) \mathrm{d} \tau, \omega_{1 r}(0)=0,
\end{aligned}
$$

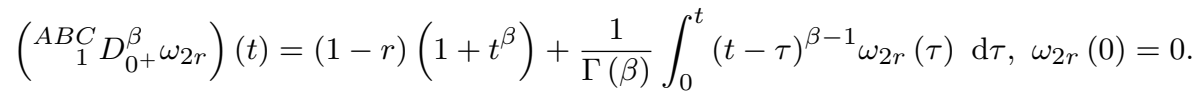

If $\beta=1$, then the exact solution of system (5.2) is $\omega(t)=\eta\left(e^{t}-1\right)$.

- For $\underset{g H}{A B C}[(2)-\beta]$-differentiability, the Volterra FFIDE (5.1) will be equivalent to the underlying crisp system of Volterra FIDEs:

$$
\begin{aligned}
& \left(\underset{2}{A B C} D_{0^{+}}^{\beta} \omega_{1 r}\right)(t)=(r-1)\left(1+t^{\beta}\right)+\frac{1}{\Gamma(\beta)} \int_{0}^{t}(t-\tau)^{\beta-1} \omega_{2 r}(\tau) \mathrm{d} \tau, \omega_{1 r}(0)=0 \\
& \left(\underset{2}{A B C} D_{0^{+}}^{\beta} \omega_{2 r}\right)(t)=(1-r)\left(1+t^{\beta}\right)+\frac{1}{\Gamma(\beta)} \int_{0}^{t}(t-\tau)^{\beta-1} \omega_{1 r}(\tau) \mathrm{d} \tau, \omega_{2 r}(0)=0 .
\end{aligned}
$$

If $\beta=1$, then the exact solution of system (5.3) is $\omega(t)=\eta(\sin t-\cos t+1)$.

Applying the RKHS method with $n=40$, the achieved numerical outcomes are summarized in the form of tables and graphical representations as follows. The 3D surface plot for the exact and approximate RKHS solutions and its 

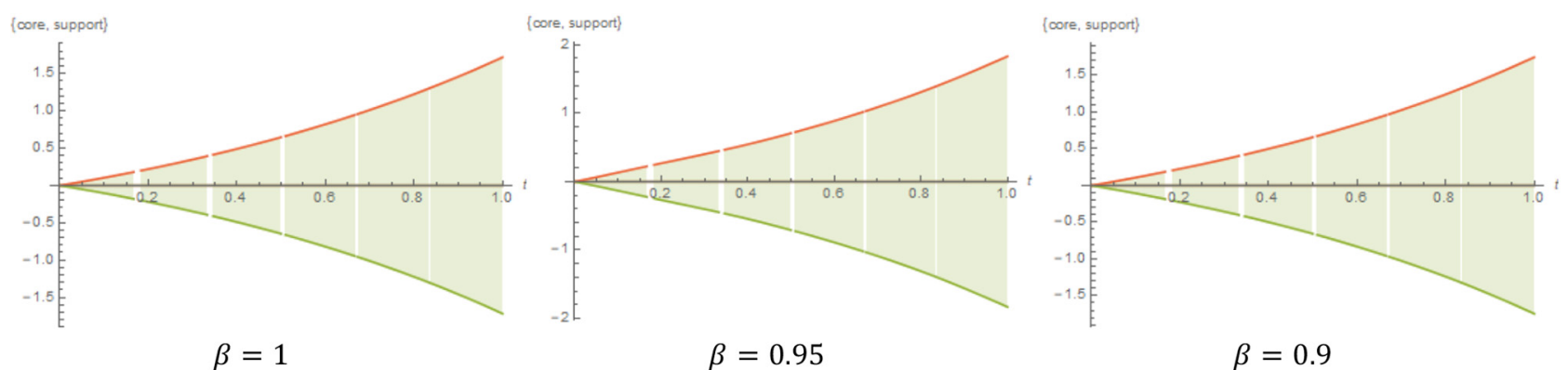

$\beta=0.95$
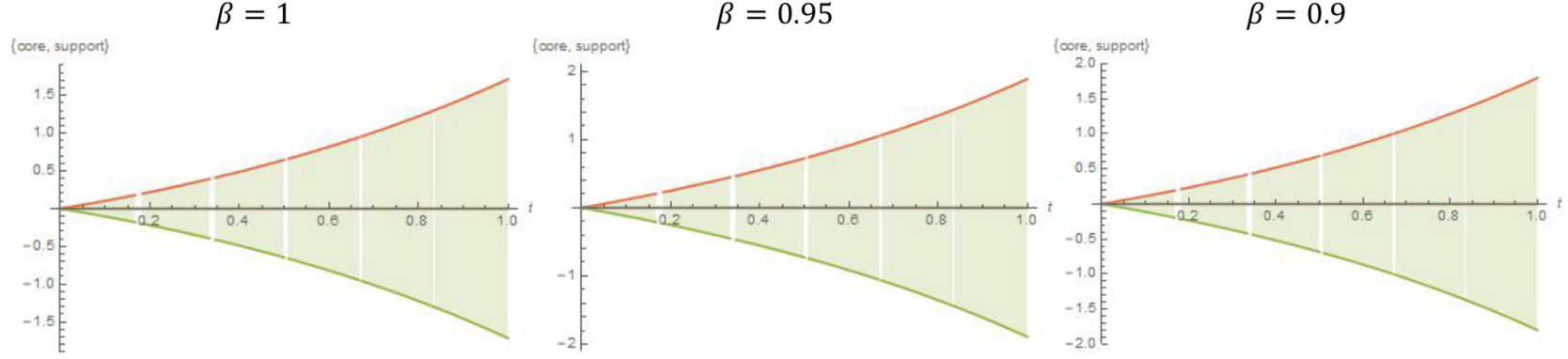

$\beta=1$

$\beta=0.95$

$\beta=0.9$

Figure 2. Approximate core and support at different $\beta$ values by means of $\underset{g H}{A B C}[(1)-\beta]$ differentiability (above) and $\underset{g H}{C}[(1)-\beta]$-differentiability (below) of Example 5.1, case 1.

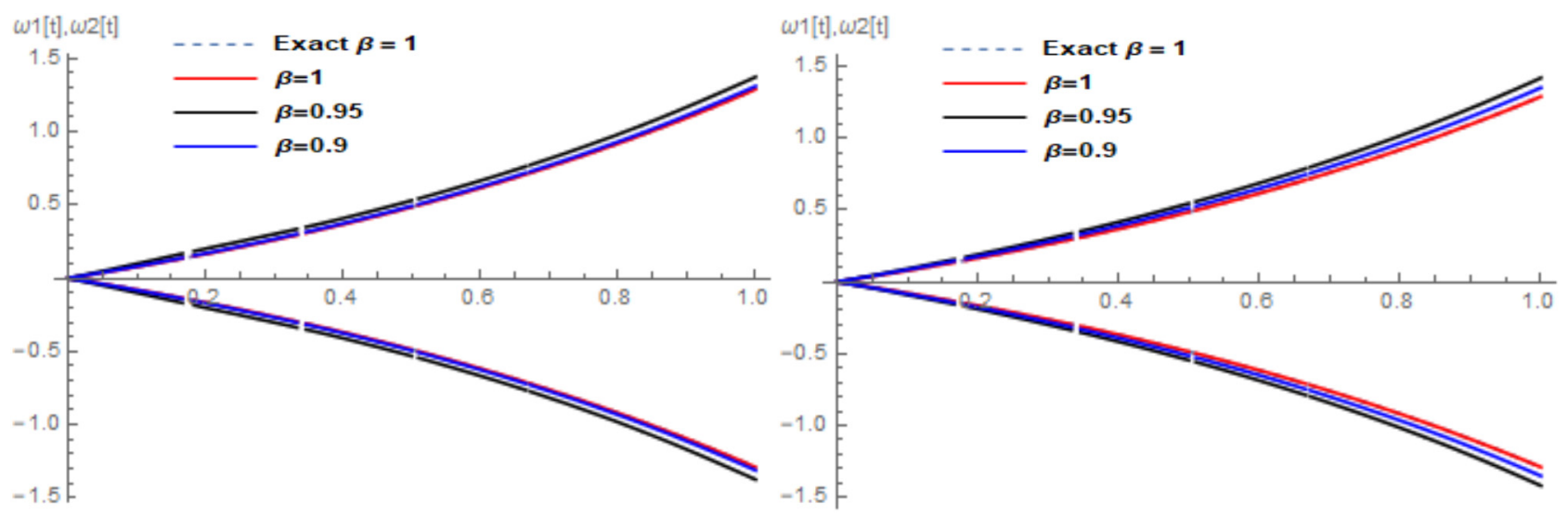

Figure 3. Graphs of $\beta$-level curves for differe nt $\beta$ and $r=0.25$ in terms of $\underset{g H}{A B C}[(1)-\beta]$ differentiability (left) and in terms of ${ }_{g H}^{C}[(1)-\beta]$-differentiability (right) of Example 5.1, case 1 .

derivatives in view of $\underset{g H}{A B C}[(1)-\beta]$-differentiable are given in Figure 1. While Figure 2 exhibits a comparison between the approximate cores and supports for the fuzzy solutions under $\underset{g H}{A B C}[(1)-\beta]$ - and $\underset{g H}{C}[(1)-\beta]$-differentiabilities at different $\beta$ indexes.

Figure 3 displays a comparison of approximate solutions for different $\beta$ indexes in the light of $\underset{g H}{A B C}[(1)-\beta]$ - and ${ }_{g H}^{C}[(1)-\beta]$-differentiabilities. In Figure 4, $r$-level curves are presented for different $r$ values with $\beta=0.9$ under gHdifferentiability in Caputo and $\mathrm{ABC}$ sense. It is clear that the surface plots of fuzzy approximate solutions are constantly dependent on corresponding fractional values. Numerical approximations of Example 5.1 compared with the exact solutions at $\beta=1$ and are summarized in Table 1 in view of $\underset{g H}{A B C}[(1)-\beta]$ - and $\underset{g H}{C}[(1)-\beta]$-differentiabilities over the 

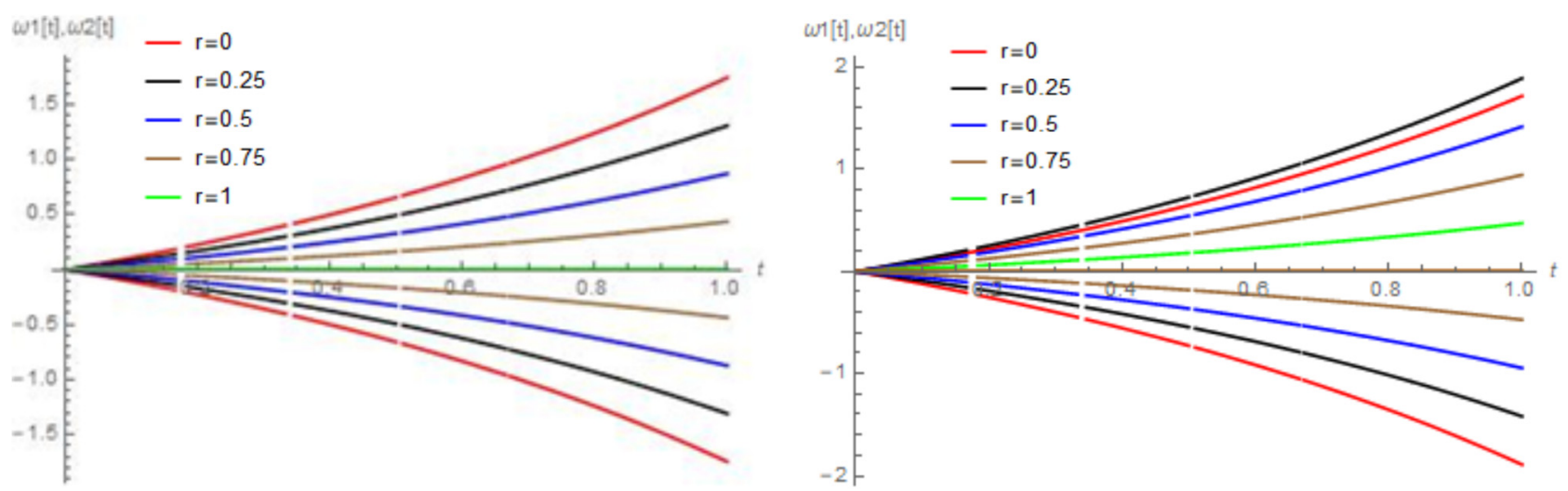

FiguRE 4. Graphs of $r$-level curves for different $r$ values at $\beta=0.9$ by means of $\underset{g H}{A B C}[(1)-\beta]$ differentiability (left) and $\underset{g H}{C}[(1)-\beta]$-differentiability (right) for Example 5.1, case 1.

TABLE 1. Numerical results of Example 5.1 in terms of $\underset{g H}{A B C}[(1)-\beta]$ - and $\underset{g H}{C}[(1)-\beta]$ differentiabilities.

\begin{tabular}{|c|c|c|c|c|c|c|}
\hline \multirow[t]{2}{*}{$t_{i}$} & \multicolumn{5}{|c|}{ Approximate RKHS solution $\omega_{1 r}(t)$ with $r=0.75$} & \multirow{2}{*}{$\begin{array}{l}\text { Absolute Error } \\
\text { (ABC operator) } \\
\beta=1\end{array}$} \\
\hline & $\beta=1$ & $A B C[(1)-0.95]$ & ${ }^{C}[(1)-0.95]$ & $A B C[(1)-0.9]$ & ${ }^{C}[(1)-0.9]$ & \\
\hline 0.1 & -0.0262927 & -0.03311720 & -0.03024964 & -0.02707321 & -0.02810861 & $1.3170346 \times 10^{-6}$ \\
\hline 0.3 & -0.0874647 & -0.10040613 & -0.09947497 & -0.08915656 & -0.09289777 & $2.1570681 \times 10^{-6}$ \\
\hline 0.5 & -0.1621800 & -0.17685462 & -0.18167977 & -0.16441221 & -0.17123478 & $1.9887312 \times 10^{-6}$ \\
\hline 0.7 & -0.2534380 & -0.27180802 & -0.28093880 & -0.25677242 & -0.26633358 & $6.9243846 \times 10^{-7}$ \\
\hline 0.9 & -0.3649010 & -0.38923951 & -0.40211111 & -0.37028170 & -0.38244686 & $1.9674212 \times 10^{-6}$ \\
\hline \multirow[t]{2}{*}{$t_{i}$} & \multicolumn{5}{|c|}{ Approximate RKHS solution $\omega_{2 r}(t)$ with $r=0.75$} & \multirow{2}{*}{$\begin{array}{l}\text { Absolute Error } \\
\text { (ABC operator) } \\
\beta=1\end{array}$} \\
\hline & $\beta=1$ & $A B C[(2)-0.95$ & ${ }^{C}[(2)-0.95]$ & $A B C[(2)-0.9]$ & ${ }^{C}[(2)-0.9]$ & \\
\hline 0.1 & 0.0262927 & 0.03311720 & 0.03024964 & 0.02707321 & 0.02810861 & $1.3170346 \times 10^{-6}$ \\
\hline 0.3 & 0.0874647 & 0.10040613 & 0.09947497 & 0.08915656 & 0.09289777 & $2.1570681 \times 10^{-6}$ \\
\hline 0.5 & 0.1621800 & 0.17685462 & 0.18167977 & 0.16441221 & 0.17123478 & $1.9887312 \times 10^{-6}$ \\
\hline 0.7 & 0.2534380 & 0.27180802 & 0.28093880 & 0.25677242 & 0.26633358 & $6.9243846 \times 10^{-7}$ \\
\hline 0.9 & 0.3649010 & 0.38923951 & 0.40211111 & 0.37028170 & 0.38244686 & $1.9674212 \times 10^{-6}$ \\
\hline
\end{tabular}

interval [0.1] with step-size 0.2. Furthermore, some representative results of $\underset{g H}{A B C}[(2)-\beta]$-differentiability are presented in Figures 5-8 and Table 2 as well. From the figures, it can be seen that all the plots are nearly compatible, analogous, and similar in behavior as well as fully consistent with each other, especially when considering the fuzzy ABC derivative of integer order $\beta=1$. Also, the solution is an interval at each instantaneous point of all levels of r-cut, which means that the solution is a fuzzy function at every point in the internal domain.

Example 5.2. Consider $H(t, \tau, \omega(\tau))=0, K(t, \tau, \omega(\tau))=-\omega, p(t)=0$, and $f(t)=-2 \eta \sin t$, along with the fuzzy initial condition $\omega(0)=\eta$, where $\eta$ is the generalized triangular fuzzy number $(-2,1,2 ; 1)$. Herein, the FFIDE (1.1) converts into the underlying Volterra FFIDE:

$$
\left(\underset{g H}{A B C} D_{0^{+}}^{\beta} \omega\right)(t)=-2 \eta \sin t-\int_{0}^{t} \omega(\tau) \mathrm{d} \tau, \omega(0)=\eta, 0<\beta \leq 1 .
$$



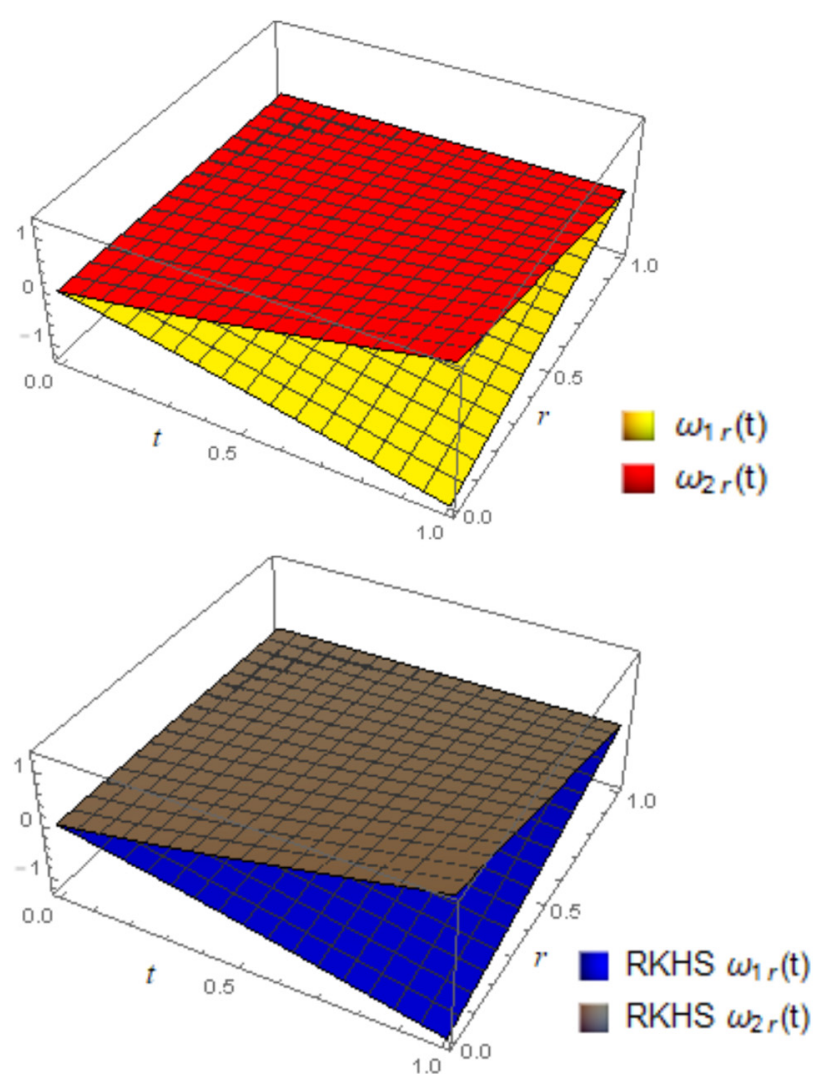
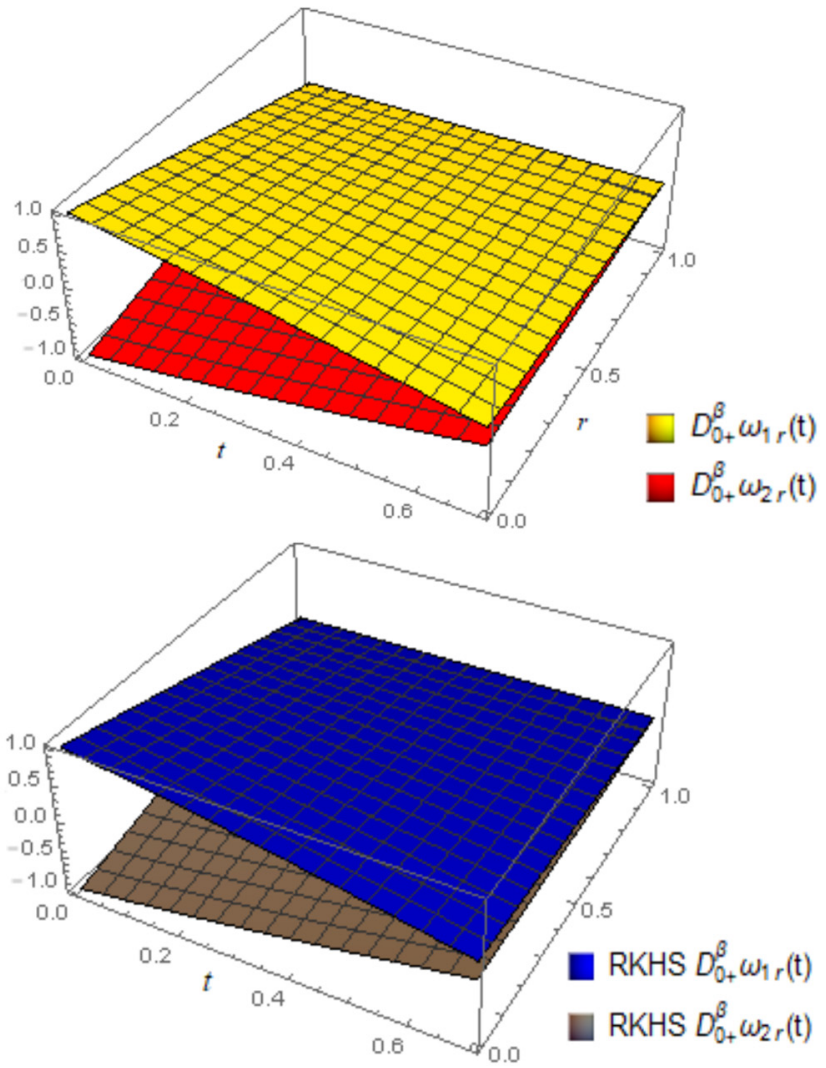

FiguRE 5. Comparison between the exact solution with its derivative (above) and approximate RKHS-solution (below) at $\beta=1$ by means of $\underset{g H}{A B C}[(2)-\beta]$-differentiability for Example 5.1, case 2 .

TABLE 2. Numerical results for Example 5.1 in terms of $\underset{g H}{A B C}[(2)-\beta]$ - and $\underset{g H}{C}[(2)-\beta]$ differentiabilities.

\begin{tabular}{|c|c|c|c|c|c|c|}
\hline \multirow[t]{2}{*}{$t_{i}$} & \multicolumn{5}{|c|}{ Approximate RKHS solution $\omega_{1 r}(t)$ with $r=0.75$} & \multirow{2}{*}{$\begin{array}{l}\text { Absolute Error } \\
\text { (ABC operator) } \\
\beta=1\end{array}$} \\
\hline & $\beta=1$ & $A B C[(1)-0.95$ & ${ }^{C}[(1)-0.95]$ & ${ }^{A B C}[(1)-0.9]$ & ${ }^{C}[(1)-0.9]$ & \\
\hline 0.1 & -0.02620800 & -0.03393330 & -0.03018032 & -0.027490546 & -0.02807284 & $6.3902269 \times 10^{-7}$ \\
\hline 0.3 & -0.08504360 & -0.09547365 & -0.09679080 & -0.086671534 & -0.09095203 & $2.3318557 \times 10^{-6}$ \\
\hline 0.5 & -0.15045516 & -0.15424662 & -0.16366358 & -0.149360350 & -0.15726918 & $5.5832508 \times 10^{-6}$ \\
\hline 0.7 & -0.21983502 & -0.21543716 & -0.23015837 & -0.215064637 & -0.22536286 & $8.8530113 \times 10^{-6}$ \\
\hline \multirow[t]{2}{*}{0.9} & -0.29041737 & -0.27599123 & -0.29427415 & -0.281029870 & -0.29283396 & $1.1868656 \times 10^{-5}$ \\
\hline & \multicolumn{5}{|c|}{ Approximate RKHS solution $\omega_{2 r}(t)$ with $r=0.75$} & Absolute Error \\
\hline$t_{i}$ & $\beta=1$ & $A B C[(2)-0.95$ & ${ }^{C}[(2)-0.95]$ & $A B C[(2)-0.9]$ & ${ }^{C}[(2)-0.9]$ & $\begin{array}{l}\text { (ABC operator) } \\
\beta=1\end{array}$ \\
\hline 0.1 & 0.02620800 & 0.03393330 & 0.03018032 & 0.027490546 & 0.02807284 & $6.3902269 \times 10^{-7}$ \\
\hline 0.3 & 0.08504360 & 0.09547365 & 0.09679080 & 0.086671534 & 0.09095203 & $2.3318557 \times 10^{-6}$ \\
\hline 0.5 & 0.15045516 & 0.15424662 & 0.16366358 & 0.149360350 & 0.15726918 & $5.5832508 \times 10^{-6}$ \\
\hline 0.7 & 0.21983502 & 0.21543716 & 0.23015837 & 0.215064637 & 0.22536286 & $8.8530113 \times 10^{-6}$ \\
\hline 0.9 & 0.29041737 & 0.27599123 & 0.29427415 & 0.281029870 & 0.29283396 & $1.1868656 \times 10^{-5}$ \\
\hline
\end{tabular}




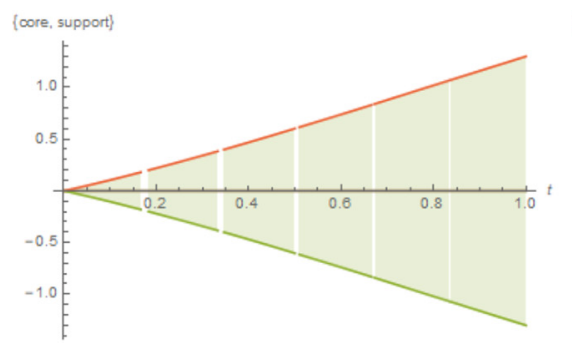

$\beta=1$

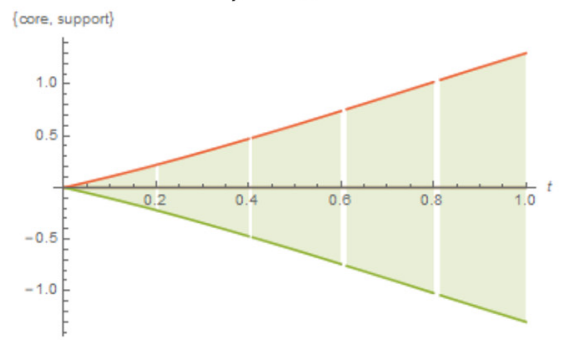

$\beta=1$

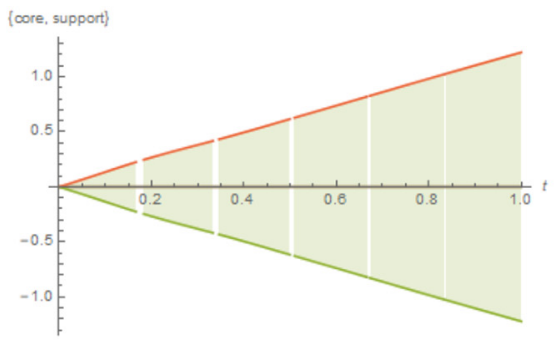

$\beta=0.95$

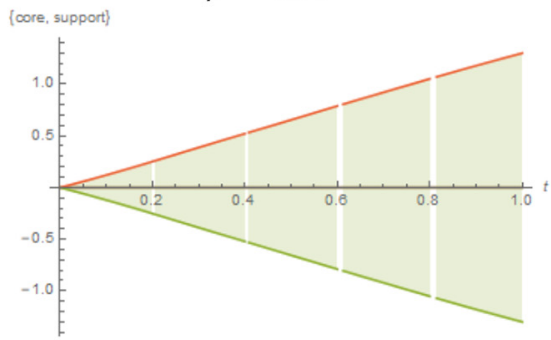

$\beta=0.95$

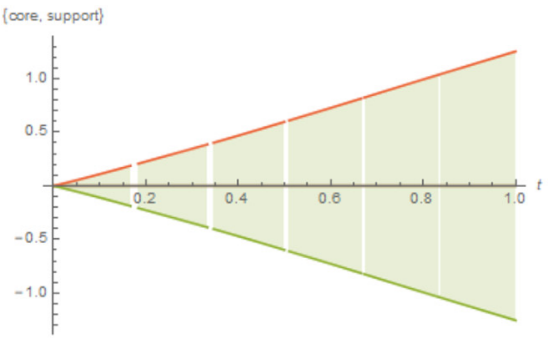

$\beta=0.9$

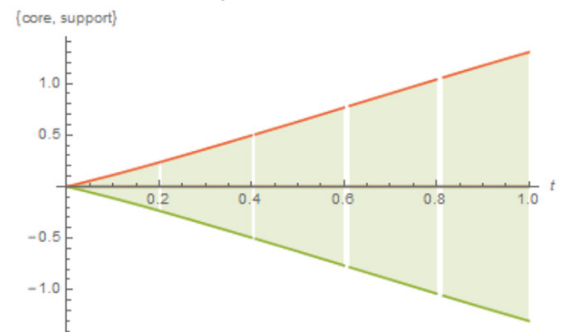

$\beta=0.9$

Figure 6. Approximate core and support for different $\beta$ values in view of $\underset{g H}{A B C}[(2)-\beta]$ differentiability (above) and in view of ${ }_{g H}^{C}[(2)-\beta]$-differentiability (below) of Example 5.1, case 2 .
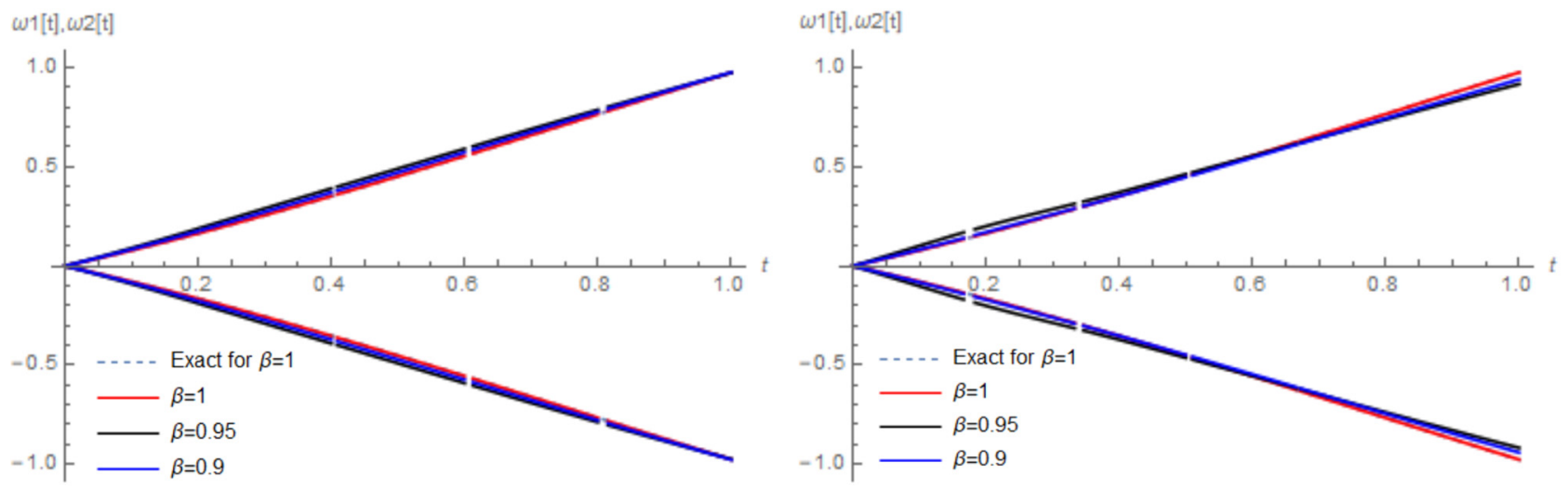

FiguRE 7. Graphs of $\beta$-level curves for different $\beta$ indexes with $r=0.25$ in terms of $\underset{g H}{A B C}[(2)-\beta]$-differentiability (left) and $\underset{g H}{C}[(2)-\beta]$-differentiability (right) for Example 5.1, case 2 .

- For $\underset{g H}{A B C}[(1)-\beta]$-differentiability, the Volterra FFIDE (5.4) will be equivalent to the underlying crisp system of Volterra FIDEs:

$$
\begin{aligned}
& \left(\begin{array}{c}
A B C \\
{ }_{1} D_{0^{+}}^{\beta} \omega_{1 r}
\end{array}\right)(t)=(2 r-4) \sin t-\int_{0}^{t} \omega_{2 r}(\tau) \mathrm{d} \tau, \omega_{1 r}(0)=3 r-2, \\
& \left(\begin{array}{c}
A B C \\
1
\end{array} D_{0^{+}}^{\beta} \omega_{2 r}\right)(t)=(4-6 r) \sin t-\int_{0}^{t} \omega_{1 r}(\tau) \mathrm{d} \tau, \omega_{2 r}(0)=2-r .
\end{aligned}
$$



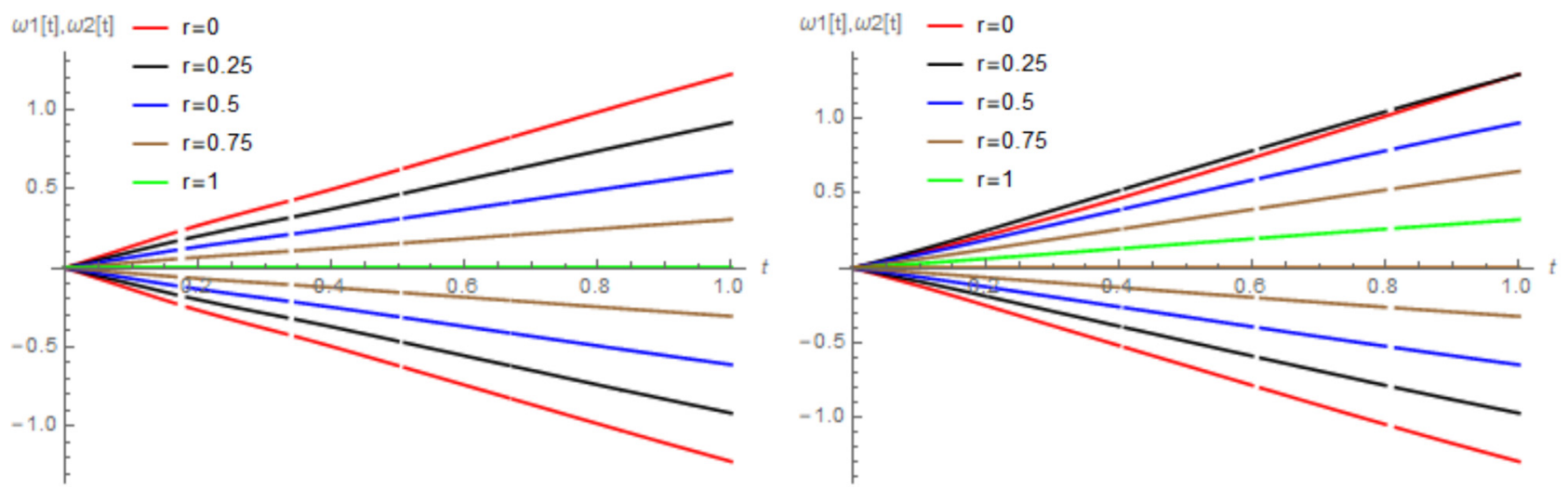

Figure 8. Graphs of $r$-level curves for different $r$ values at $\beta=0.9$ under $\underset{g H}{A B C}[(2)-\beta]$ differentiability (left) and under $\underset{g H}{C}[(2)-\beta]$-differentiability (right) for Example 5.1, case 2.

If $\beta=1$, then the $r$-cut representations of exact solution of system (5.5) are given as

$$
\begin{gathered}
\omega_{1 r}(t)=-r t \sin t+(2-r) \cos t+4(r-1) \cosh t, \\
\omega_{2 r}(t)=-r t \sin t+(3 r-2) \cos t-4(r-1) \cosh t
\end{gathered}
$$

- For $\underset{g H}{A B C}[(2)-\beta]$-differentiability, the Volterra FFIDE (5.4) will be equivalent to the underlying crisp system of Volterra FIDEs:

$$
\begin{aligned}
& \left(\underset{2}{A B C} D_{0^{+}}^{\beta} \omega_{1 r}\right)(t)=(4-6 r) \sin t-\int_{0}^{t} \omega_{1 r}(\tau) \mathrm{d} \tau, \omega_{1 r}(0)=3 r-2, \\
& \left(\underset{2}{A B C} D_{0^{+}}^{\beta} \omega_{2 r}\right)(t)=(2 r-4) \sin t-\int_{0}^{t} \omega_{2 r}(\tau) \mathrm{d} \tau, \omega_{2 r}(0)=2-r .
\end{aligned}
$$

If $\beta=1$, then the exact solution of system (5.6) is $\omega(t)=\eta(\cos t-t \sin t)$.

Applying the RKHS method with $n=15$, numerical and graphical results for fuzzy approximate solutions in terms of $\underset{g H}{A B C}[(1)-\beta]$ - and $\underset{g H}{C}[(1)-\beta]$-differentiablities are shown in Figures 9 and 10 and in terms of $\begin{gathered}A B C \\ g H\end{gathered}[(2)-\beta]$ - and ${ }_{g H}^{C}[(2)-\beta]$-differentiablities are shown in Figures 11 and 12.

Example 5.3. Consider $\beta=\frac{1}{2}, \quad H(t, \tau, \omega(\tau))=p(t)=0, \quad K(t, \tau, \omega(\tau))=\frac{-1}{3} \tau t \omega(\tau), \quad$ and $f(t)=\eta \zeta(t)$, where $\eta$ is the generalized triangular fuzzy number $(1,2,5 ; 1)$, and $\zeta(t)=\frac{1}{15 \sqrt{\pi}} 24 \sqrt{\mathrm{t}}(15+2 t(5+2 t))-$ $\left.\sqrt{\pi}\left(180+t\left(180+90 t+t^{5}\right)\right)+180 e^{t} \sqrt{\pi} \operatorname{Erfc}(\mathrm{z})\right)$, in which $\operatorname{Erfc}(\mathrm{z})$ refers to the complementary error function, equipped with the fuzzy initial condition $\omega(0)=0$. Herein, the FFIDE (1.1) converts into the underlying Volterra FFIDE:

$$
\left(\underset{g H}{A B C} D_{0^{+}}^{\frac{1}{2}} \omega\right)(t)=\eta \zeta(t)-\frac{1}{3} \int_{0}^{t} \tau t \omega(\tau) \mathrm{d} \tau, \omega(0)=0
$$

By means of $\underset{g H}{A B C}[(1)-\beta]$-differentiability, the Volterra FFIDE (5.7) will be equivalent to the underlying crisp system of Volterra FIDEs:

$$
\begin{aligned}
& \left(\begin{array}{c}
A B C \\
{ }_{1}
\end{array} D_{0^{+}}^{\frac{1}{2}} \omega_{1 r}\right)(t)=(r+1) \zeta(t)-\frac{1}{3} \int_{0}^{t} \tau t \omega_{2 r}(\tau) \mathrm{d} \tau, \omega_{1 r}(0)=0, \\
& \left(\begin{array}{c}
A B C \\
1
\end{array} D_{0^{+}}^{\frac{1}{2}} \omega_{2 r}\right)(t)=(5-3 r) \zeta(t)-\frac{1}{3} \int_{0}^{t} \tau t \omega_{1 r}(\tau) \mathrm{d} \tau, \omega_{2 r}(0)=0,
\end{aligned}
$$



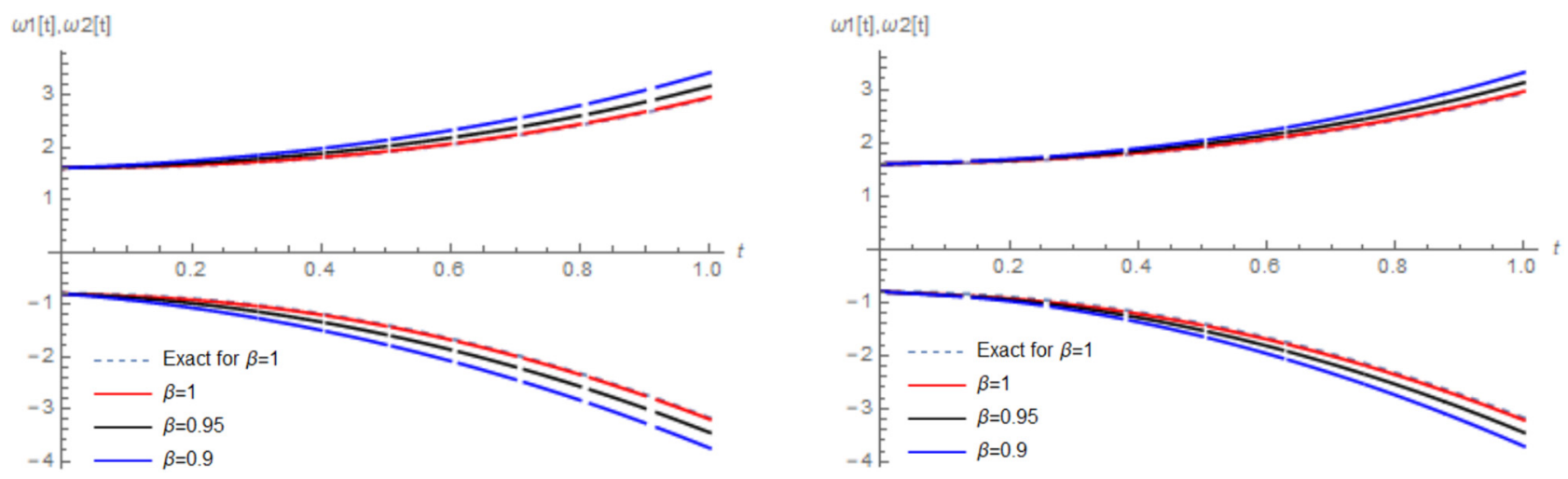

Figure 9. Graphs of $\beta$-level curves for different $\beta$ indexes at $r=0.4$ in terms of $\underset{g H}{A B C}[(1)-\beta]$ differentiability (left) and in terms of ${ }_{g H}^{C}[(1)-\beta]$-differentiability (right) for Example 5.2, case 1 .
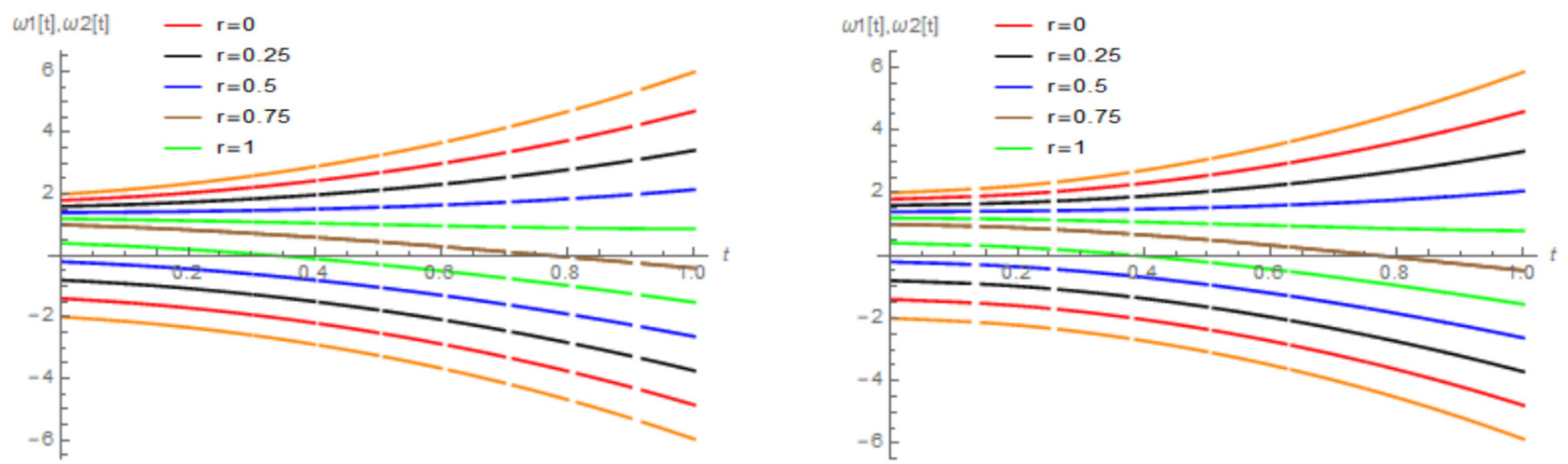

Figure 10. Graphs of $r$-level curves for different $r$ values at $\beta=0.8$ under $\underset{g H}{A B C}[(1)-\beta]$ differentiability (left) and under $\underset{g H}{C}[(1)-\beta]$-differentiability $($ right $)$ for Example 5.2, case 1.
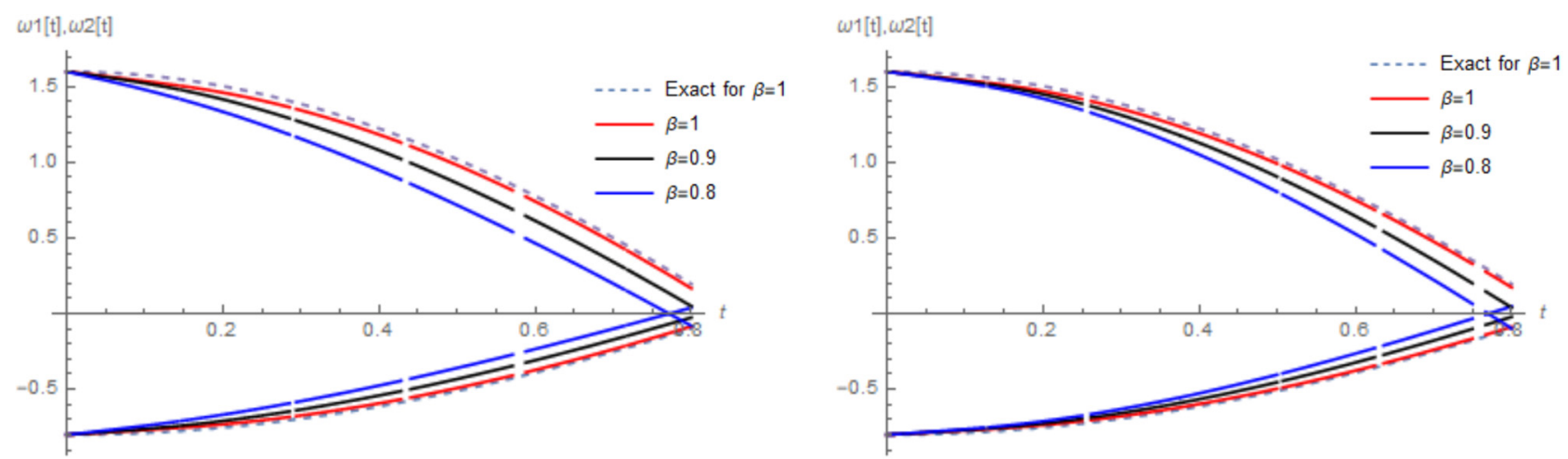

Figure 11. Graphs of $\beta$-level curves for different $\beta$ indexes at $r=0.4$ in terms of $\underset{g H}{A B C}[(2)-\beta]$ differentiability (left) and in terms of ${ }_{g H}^{C}[(2)-\beta]$-differentiability (right) for Example 5.2, case 2 . 


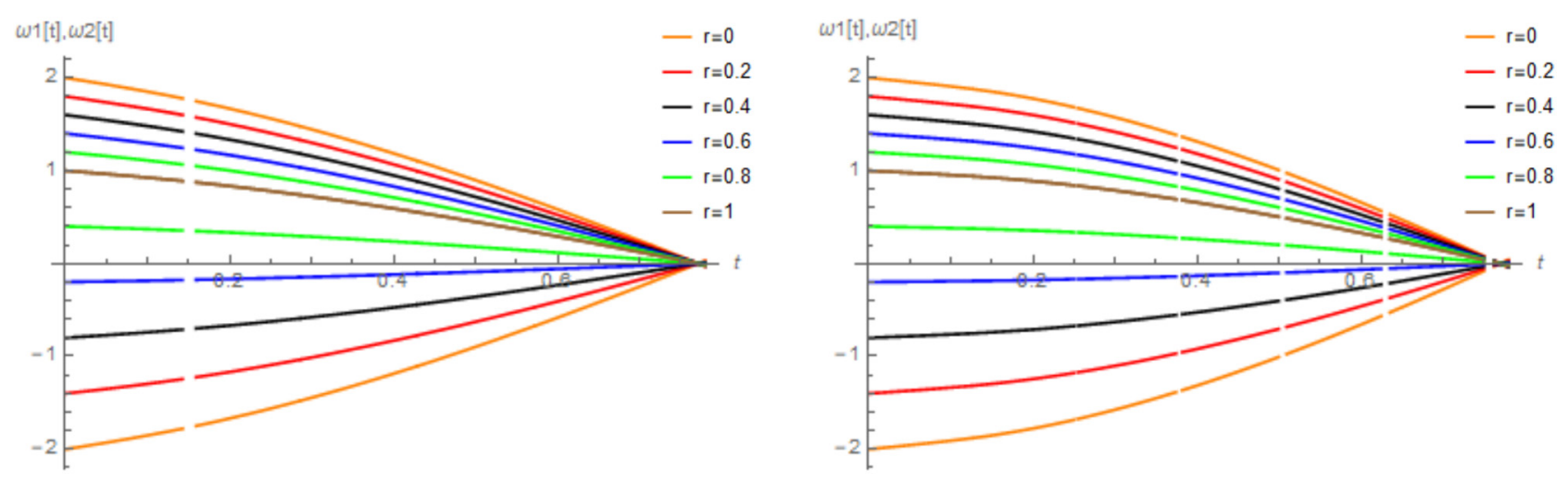

Figure 12. Graphs of $r$-level curves for different $r$ values at $\beta=0.8$ under $\underset{g H}{A B C}[(2)-\beta]$ differentiability (left) and under $\underset{g H}{C}[(2)-\beta]$-differentiability (right) for Example 5.2, case 2.

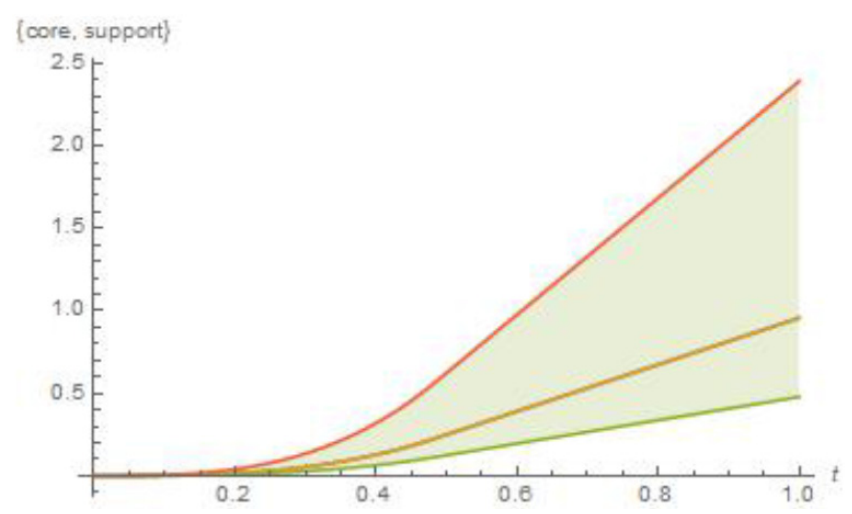

FiguRE 13. Approximate core and support $\underset{g H}{A B C}[(1)-0.5]$-differentiability of Example 5.3.

whose exact solution is $\omega(\mathrm{t})=\eta t^{3}$. Using the RKHSM with $n=25$, numerical results for Example 5.3 are given in Table 3 and Figures 15 and 16 in order to show the accuracy of the proposed method and to support the theoretical framework as well.

Example 5.4. Consider $\beta=\frac{1}{2}, \quad K(t, \tau, \omega(\tau))=p(t)=0, \quad H(t, \tau, \omega(\tau))=\frac{1}{10} \tau^{2} \quad t \quad \omega^{2}(\tau)$, and $f(t)=$ $\left[f_{1}, f_{2}\right]$, where $f_{1}(t)=-\frac{1}{40} r^{2} t^{2}+2 r\left(-1+\frac{2 \sqrt{t}}{\sqrt{\pi}}+e^{t} \operatorname{Erfc}[\sqrt{t}]\right)$ and $f_{2}(t)=\frac{1}{40 \sqrt{\pi}}(r-2)\left(\left(160 \sqrt{t}+\sqrt{\pi}\left(-80+(-2+r) t^{2}\right)\right.\right.$ $\left.\left.+80 e^{t} \sqrt{\pi} \operatorname{Erfc}[\sqrt{t}]\right)\right)$ along with the fuzzy initial condition $\omega(0)=0$, Herein, the FFIDE (1.1) converts into the underlying nonlinear Fredholm FFIDE:

$$
\left(\underset{g H C}{A B C} D_{0^{+}}^{\frac{1}{2}} \omega\right)(t)=f(t)+\frac{1}{10} \int_{0}^{1}\left(\tau^{2} t\right)(\omega(\tau))^{2} \mathrm{~d} \tau, \omega(0)=0
$$

By means of $\underset{g H}{A B C}[(1)-\beta]$-differentiability, the Fredholm FFIDE (5.9) will be equivalent to the underlying nonlinear crisp system of Fredholm FIDEs:

$$
\left({ }_{1}^{A B C} D_{0^{+}}^{\frac{1}{2}} \omega_{1 r}\right)(t)=f_{1}(t)+\frac{1}{10} \int_{0}^{1}\left(\tau^{2} t\right)\left(\omega_{1 r}(\tau)\right)^{2} \mathrm{~d} \tau, \omega_{1 r}(0)=0
$$


TABLE 3. Numerical results of Example 5.3 under $\underset{g H}{A B C}[(1)-\beta]$-differentiability.

\begin{tabular}{ccccc}
\hline$t_{k}$ & $r$ & $\omega_{1 r}(t)$ & $\omega_{2 r}(t)$ & Absolute Error \\
\hline 0.2 & & 0.0080000107 & 0.0400000535 & $1.0695 \times 10^{-8}$ \\
0.4 & & 0.0640000186 & 0.3200000928 & $1.8557 \times 10^{-8}$ \\
0.6 & 0.0 & 0.2160000273 & 1.0800001360 & $2.7263 \times 10^{-8}$ \\
0.8 & & 0.5120001372 & 2.5600006860 & $1.3721 \times 10^{-7}$ \\
\hline 0.2 & & 0.0096000128 & 0.0352000471 & $1.2834 \times 10^{-8}$ \\
0.4 & & 0.0768000223 & 0.2816000816 & $2.2268 \times 10^{-8}$ \\
0.6 & 0.2 & 0.2592000327 & 0.9504001200 & $3.2716 \times 10^{-8}$ \\
0.8 & & 0.6144001646 & 2.2528006040 & $1.6465 \times 10^{-8}$ \\
\hline 0.2 & & 0.0112000149 & 0.0304000406 & $1.4973 \times 10^{-8}$ \\
0.4 & & 0.0896000259 & 0.2432000705 & $2.5979 \times 10^{-8}$ \\
0.6 & 0.4 & 0.3024000382 & 0.8208001036 & $3.8168 \times 10^{-8}$ \\
0.8 & & 0.7168001921 & 1.9456005210 & $1.9209 \times 10^{-8}$ \\
\hline 0.2 & & 0.0128000171 & 0.0256000342 & $1.7112 \times 10^{-8}$ \\
0.4 & & 0.1024000297 & 0.2048000594 & $2.9691 \times 10^{-8}$ \\
0.6 & 0.6 & 0.3456000436 & 0.6912000872 & $4.3621 \times 10^{-8}$ \\
0.8 & & 0.8192002195 & 1.6384004390 & $2.1953 \times 10^{-7}$ \\
\hline 0.2 & & 0.0144000193 & 0.0208000278 & $1.9251 \times 10^{-8}$ \\
0.4 & & 0.1152000334 & 0.1664000482 & $3.3402 \times 10^{-8}$ \\
0.6 & 0.8 & 0.3888000491 & 0.5616000709 & $4.9074 \times 10^{-8}$ \\
0.8 & & 0.9216002470 & 1.3312003570 & $2.4697 \times 10^{-7}$ \\
\hline 0.2 & & 0.0160000214 & 0.0160000214 & $2.1391 \times 10^{-8}$ \\
0.4 & & 0.1280000371 & 0.1280000371 & $3.7113 \times 10^{-8}$ \\
0.6 & 1.0 & 0.4320000545 & 0.4320000545 & $5.4526 \times 10^{-8}$ \\
0.8 & & 1.0240002740 & 1.0240002740 & $2.7441 \times 10^{-7}$ \\
\hline & & & & \\
$A B C$ & & & \\
1 & $\left.D_{0}^{\frac{1}{2}} \omega_{1 r}\right)$ & $(t)=f_{2}(t)+\frac{1}{10} \int_{0}^{1}\left(\tau^{2} t\right)\left(\omega_{2 r}(\tau)\right)^{2} \mathrm{~d} \tau, \omega_{2 r}(0)=0$ \\
& & & &
\end{tabular}

whose exact solution is $\omega(\mathrm{t})=(r, 2-r) t$. Using the RKHSM with $n=15$, the graphical results are reported in Figures 14 and 15 .

Example 5.5. Consider $K(t, \tau, \omega(\tau))=t, \quad H(t, \tau, \omega(\tau))=\tau, \quad p(t)=-2 e^{t}$, and $f(t)=\sinh (t)(1-t)$ $\left.+e^{2 t}+e^{-1}\right) \eta$, where $\eta$ is a fuzzy number with $[\eta]^{r}=[-\sqrt{1-r}, \sqrt{1-r}]$, equipped with the initial condition $\omega(0)=$ $0,0<\beta \leq 1, t \in[0,1]$. Herein, the FFIDE (1.1) converts into the underlying Volterra-Fredholm FFIDE:

$$
\left(\underset{g H}{A B C} D_{0^{+}}^{\beta} \omega\right)(t)+2 e^{t} \omega(t)=\left(\sinh (t)(1-t)+e^{2 t}+e^{-1}\right) \alpha+\int_{0}^{1} \tau \omega(\tau) \mathrm{d} \tau+\int_{0}^{t} t \omega(\tau) \mathrm{d} \tau .
$$

In terms of $\underset{g H}{A B C}[(1)-\beta]$-differentiability, the FFIDE (5.10) will be equivalent to the underlying crisp system of Volterra Fredholm FIDEs:

$$
\begin{aligned}
& \left(\begin{array}{c}
A B C \\
1
\end{array} D_{0^{+}}^{\beta} \omega_{1 r}\right)(t)=-2 e^{t} \omega_{1 r}(t)-\left(\sinh (t)(1-t)+e^{2 t}+e^{-1}\right) \sqrt{1-r}+\int_{0}^{1} \tau \omega_{1 r}(\tau) \mathrm{d} \tau \\
& +\int_{0}^{t} t \omega_{1 r}(\tau) \mathrm{d} \tau, \omega_{1 r}(0)=-\sqrt{1-r}
\end{aligned}
$$

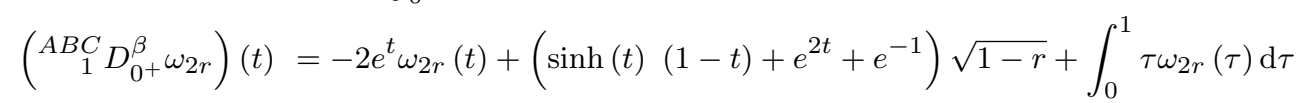



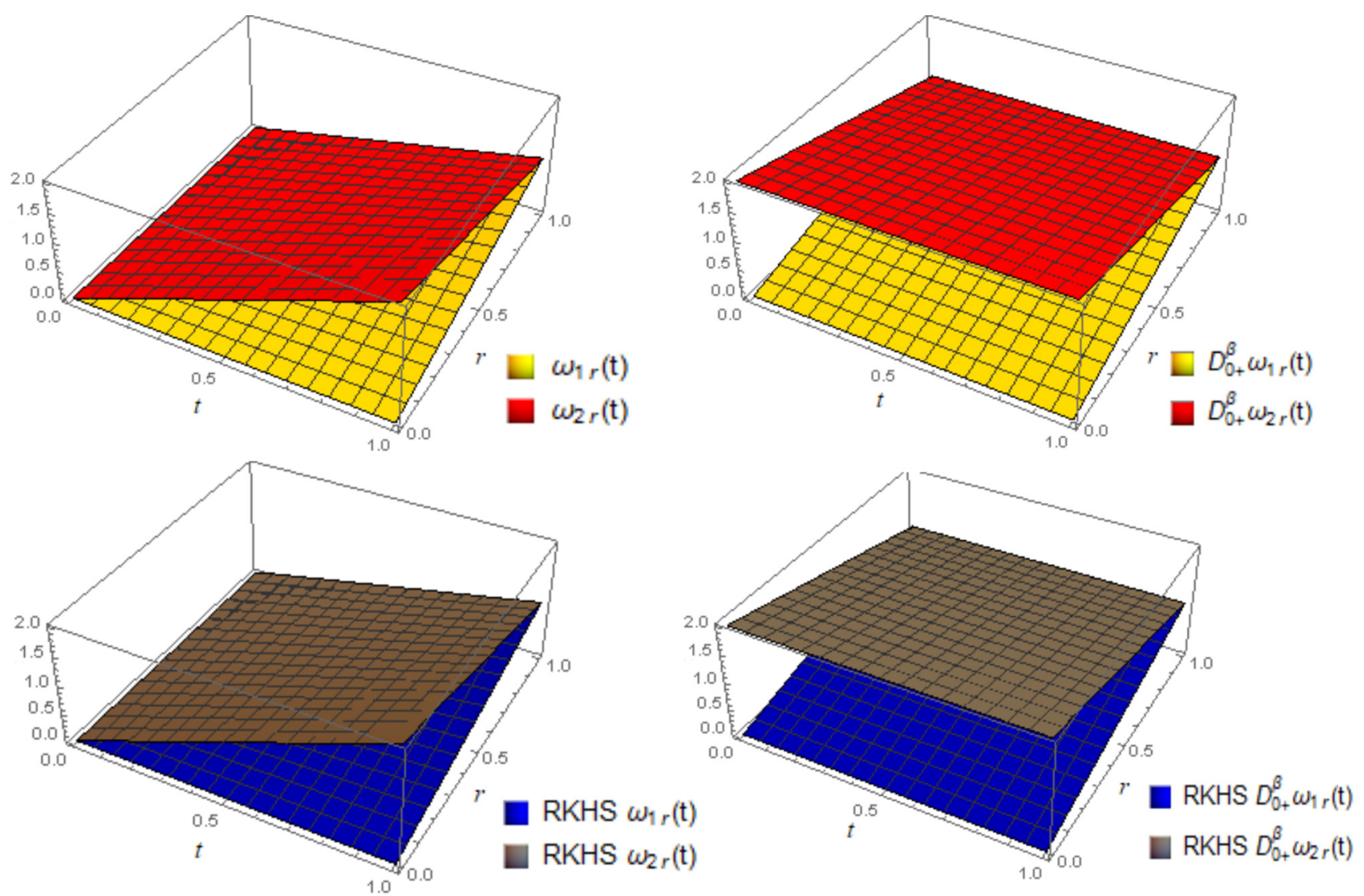

FIgURE 14. Comparison between the exact solution with its derivative (above) and the approximate RKHS-solution (below) in terms of $\underset{g H}{A B C}[(1)-0.5]$-differentiability for Example 5.4.

TABLE 4. Absolute errors of Example 5.5 in terms of $\underset{g H}{A B C}[(1)-\beta]$-differentiability with $\beta=1$.

\begin{tabular}{cccccc}
\hline$t_{k}$ & $r=0$ & $r=0.25$ & $r=0.5$ & $r=0.75$ & $r=1$ \\
\hline 0.1 & $1.6009667 \times 10^{-7}$ & $1.3864778 \times 10^{-7}$ & $1.1320544 \times 10^{-8}$ & $8.0048335 \times 10^{-8}$ & 0.00 \\
0.3 & $1.0607738 \times 10^{-7}$ & $7.4350777 \times 10^{-7}$ & $7.5008040 \times 10^{-8}$ & $5.3038693 \times 10^{-8}$ & 0.00 \\
0.5 & $7.0289852 \times 10^{-7}$ & $5.1089475 \times 10^{-7}$ & $4.9702431 \times 10^{-7}$ & $3.5144926 \times 10^{-8}$ & 0.00 \\
0.7 & $5.2261785 \times 10^{-7}$ & $4.2906646 \times 10^{-7}$ & $3.6954662 \times 10^{-7}$ & $2.6130892 \times 10^{-8}$ & 0.00 \\
0.9 & $5.2615182 \times 10^{-7}$ & $5.3071953 \times 10^{-6}$ & $3.7204551 \times 10^{-7}$ & $2.6307591 \times 10^{-7}$ & 0.00 \\
\hline
\end{tabular}

$$
+\int_{0}^{t} t \omega_{2 r}(\tau) \mathrm{d} \tau, \omega_{2 r}(0)=\sqrt{1-r}
$$

whose exact solution for $\beta=1$ is $\omega(t)=\eta \cosh (t)$.

Using this concept, Table 4 summarizes the error in approximating using 30 iterations in view of the RKHSM, while Figure 16 shows the core of fuzzy approximate solution for different values of $\mathrm{ABC}$ gH-derivatives such that $\beta \in\{1,0.95,0.9\}$.

Example 5.6. Consider $\beta=\frac{1}{2}, K(t, \tau, \omega(\tau))=3 \omega(\tau), p(t)=0, H(t, \tau, \omega(\tau))=t^{2} \omega(\tau)$, and $f(t)=\gamma \zeta(t)$, where $\gamma$ is the generalized triangular fuzzy number $(0,1,2 ; 1)$ and $\zeta(t)=\left(\frac{1}{35 \sqrt{\pi}}(8 \sqrt{t}(525+2 t(175+8 t(7+2 t)))-7 \sqrt{\pi}(300+\right.$ 


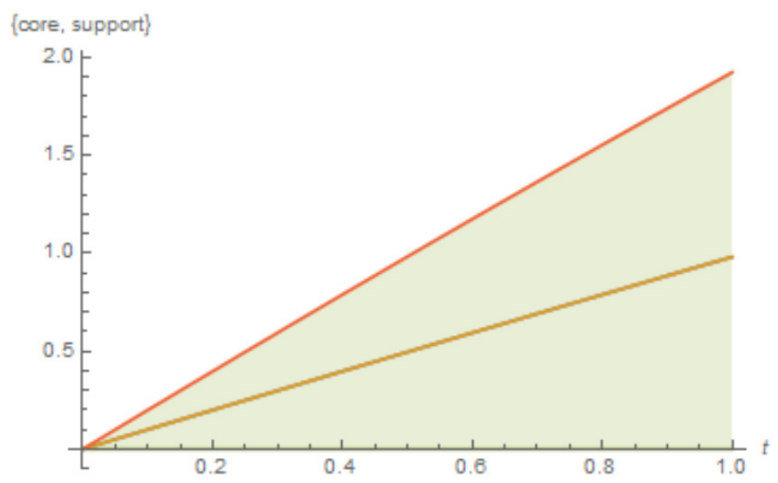

(a)

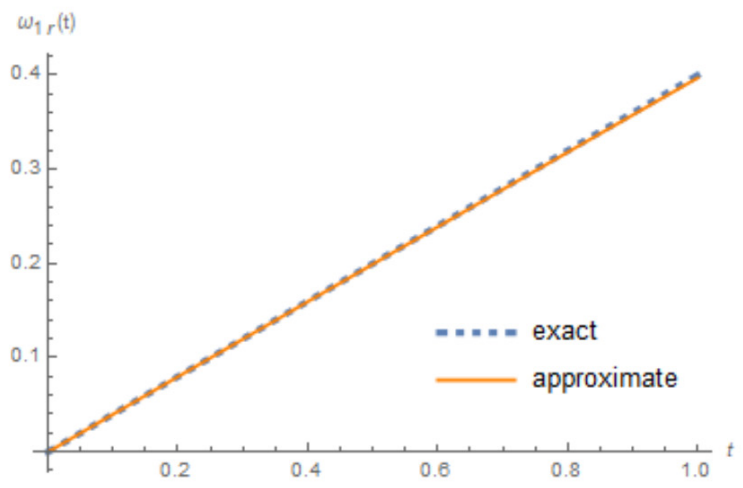

(c)

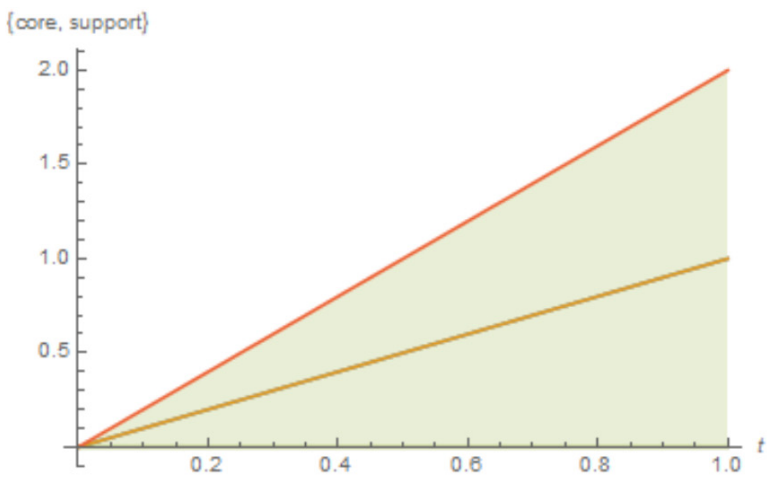

(b)

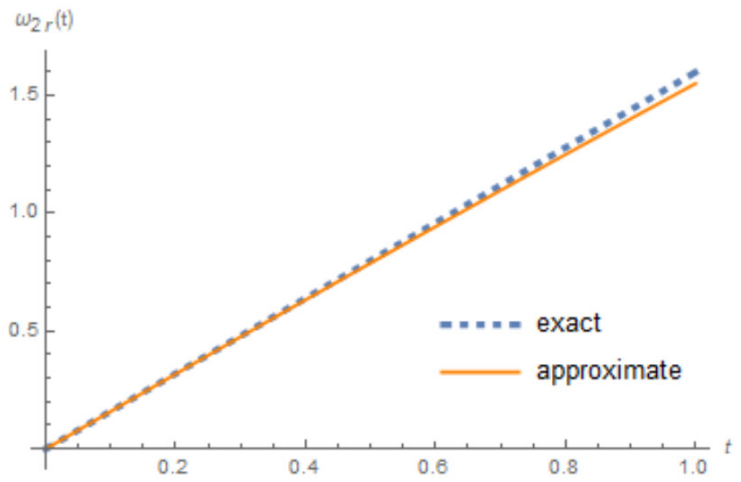

(d)

FiguRE 15. Graph of exact and approximate solutions: (a) core and support of exact, (b) core and support of RKHS; (c) upper solutions, (d) lower solutions at $r=0.4$ under $\underset{g H}{A B C}[(1)-0.5]$ differentiability of Example 5.4.
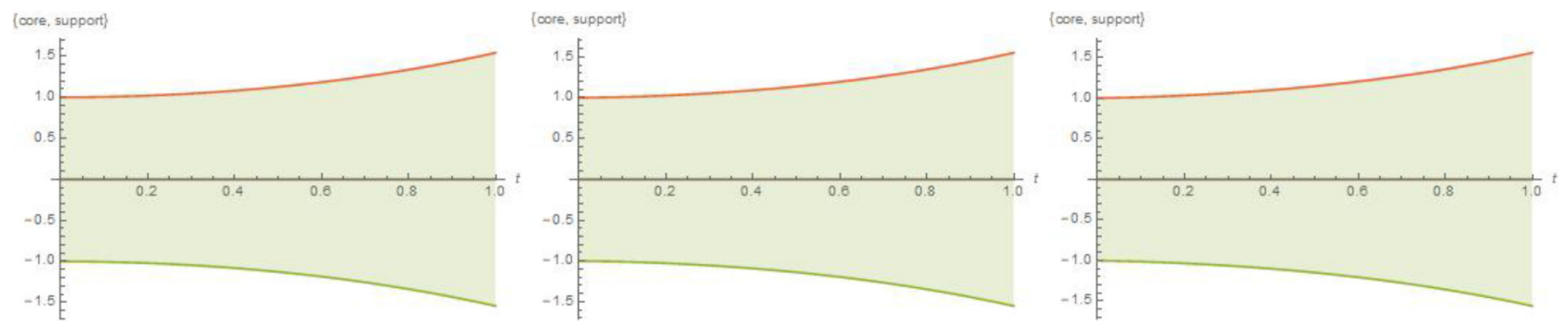

Figure 16. The cores and supports for fuzzy RKHS-solution of Example 5.5 under $\underset{g H}{A B C}[(1)-\beta]$-differentiability with $\beta=1,0.95,0.9$, respectively.

$\left.\left.\left.t\left(300+t\left(126+55 t+3 t^{3}\right)\right)\right)+2100 e^{t} \sqrt{\pi} \operatorname{Erfc}[\sqrt{t}]\right)\right)$, along with the initial condition $\omega(0)=0$. Herein, the FFIDE (1.1) converts into the underlying mixed Volterra-Fredholm FFIDE:

$$
\left(\underset{g H}{A B C} D_{0^{+}}^{\frac{1}{2}} \omega\right)(t)=\gamma \zeta(t)+\int_{0}^{1} t^{2} \omega(\tau) \mathrm{d} \tau+3 \int_{0}^{\mathrm{t}} \omega(\tau) \mathrm{d} \tau, \omega(0)=0
$$


TABLE 5. Numeric results of Example 5.6 in terms of $\underset{g H}{A B C}[(1)-0.5]$.

\begin{tabular}{ccccc}
\hline$t_{k}$ & $r$ & $\omega_{1 r}(t)$ & $\omega_{2 r}(t)$ & Absolute Error \\
\hline 0.2 & & 0.0 & 0.24320 & 0.0 \\
0.4 & & 0.0 & 1.01120 & 0.0 \\
0.6 & 0.0 & 0.0 & 2.41920 & 0.0 \\
0.8 & & 0.0 & 4.65920 & 0.0 \\
1.0 & & 0.0 & 8.00000 & 0.0 \\
\hline 0.2 & & 0.02432 & 0.21888 & $8.0880199 \times 10^{-9}$ \\
0.4 & & 0.10112 & 0.91008 & $1.4985391 \times 10^{-9}$ \\
0.6 & 0.2 & 0.24192 & 2.17728 & $2.7681427 \times 10^{-9}$ \\
0.8 & & 0.46592 & 4.19328 & $5.1021274 \times 10^{-8}$ \\
1.0 & & 0.80000 & 7.20000 & $1.0701321 \times 10^{-8}$ \\
\hline 0.2 & & 0.04864 & 0.19456 & $1.6176039 \times 10^{-9}$ \\
0.4 & & 0.20224 & 0.80896 & $2.9970783 \times 10^{-9}$ \\
0.6 & 0.4 & 0.48384 & 1.93536 & $5.5362855 \times 10^{-8}$ \\
0.8 & & 0.93184 & 3.72736 & $1.0204254 \times 10^{-8}$ \\
1.0 & & 1.60000 & 6.40000 & $2.1402643 \times 10^{-8}$ \\
\hline 0.2 & & 0.07296 & 0.17024 & $2.4264059 \times 10^{-9}$ \\
0.4 & & 0.30336 & 0.70784 & $4.4956175 \times 10^{-8}$ \\
0.6 & 0.6 & 0.72576 & 1.69344 & $8.3044283 \times 10^{-8}$ \\
0.8 & & 1.39776 & 3.26144 & $1.5306382 \times 10^{-8}$ \\
1.0 & & 2.40000 & 5.60000 & $3.2103965 \times 10^{-7}$ \\
\hline 0.2 & & 0.09728 & 0.14592 & $3.2352079 \times 10^{-9}$ \\
0.4 & & 0.40448 & 0.60672 & $5.9941567 \times 10^{-8}$ \\
0.6 & 0.8 & 0.96768 & 1.45152 & $1.1072571 \times 10^{-8}$ \\
0.8 & & 1.86368 & 2.79552 & $2.0408509 \times 10^{-8}$ \\
1.0 & & 3.20000 & 4.80000 & $4.2805287 \times 10^{-7}$ \\
\hline & & & &
\end{tabular}

By means of $\underset{g H}{A B C}[(1)-\beta]$-differentiability, the mixed FFIDE (5.13) will be equivalent into the underlying crisp system of Volterra-Fredholm FIDEs:

$$
\begin{gathered}
\left(\begin{array}{c}
A B C \\
1
\end{array} D_{0^{+}}^{\frac{1}{2}} \omega_{1 r}\right)(t)=r \zeta(t)+\int_{0}^{1} t^{2} \omega_{1 r}(\tau) \mathrm{d} \tau+3 \int_{0}^{\mathrm{t}} \omega_{1 r}(\tau) \mathrm{d} \tau, \omega_{1 r}(0)=0, \\
\left(\begin{array}{c}
A B C \\
{ }_{1} D_{0^{+}}^{\frac{1}{2}} \omega_{2 r}
\end{array}\right)(t)=(2-r) \zeta(t)+\int_{0}^{1} t^{2} \omega_{2 r}(\tau) \mathrm{d} \tau+3 \int_{0}^{\mathrm{t}} \omega_{2 r}(\tau) \mathrm{d} \tau, \omega_{2 r}(0)=0 .
\end{gathered}
$$

whose exact solution is $\omega(t)=(r, 2-r)\left(t^{4}+3 t^{2}\right)$. Using the RKHSM with $n=35$, the numerical outcomes of the proposed method are summarized in Table 5 for different $r$-levels.

\section{Conclusions}

In this work, a modified numerical algorithm has been profitably designed in light of RKHS method and employed to get approximate solutions of fuzzy fractional integrodifferential equations by means of Atangana-Baleanu-Caputo gHdifferentiability. In this direction, characterization theorem was established for ABC-fractional order, in which the studied fuzzy fractional model was consequently transformed into a crisp system of fractional IVPs under fuzzy ABC calculus. The analytical solutions have been given in series form of the parametric interval of $\mathrm{ABC}$ in the space $W_{2}^{2}[a, b] \oplus W_{2}^{2}[a, b]$. The Nth-term approximate solutions and its derivatives were uniformly convergent to the analytical solutions and its derivatives, respectively. The convergent analysis and error estimation of the proposed method have been discussed as 
well. Several applications for both linear and nonlinear, Fredholm-Volterra FFIDEs have been presented to demonstrate the reliability and effectiveness of the RKHS method and to support the theoretical framework. With providing numerical examples, the accuracy of the analytical results has been illustrated. From the achieved results, it can be observed that the posed method yields accurate approximate solutions. Anyhow, the numerical results of ABC gH-differentiability have been compared with those of generalized Caputo derivative. Using ABC gH-differentiability, we conclude that the presented study can be effectively utilized as an extended planner in handling many kinds of fractional issues under uncertainty arising in engineering, physics, and natural sciences. Hopefully, the current analysis will be employed in the near future to study more uncertain fractional models by means of ABC gH-differentiability of higher fractional order.

\section{REFERENCES}

[1] R. Agarwal, V. Lakshmikantham and J. Nieto, On the concept of solution for fractional differential equations with uncertainty. Nonlinear Anal. 72 (2010) 2859-2862.

[2] M. Alabedalhadi, M. Al-Smadi, S. Al-Omari, D. Baleanu and S. Momani, Structure of optical soliton solution for nonliear resonant space-time Schrödinger equation in conformable sense with full nonlinearity term. Phys. Scr. 95 (2020) 105215.

[3] M. Al-Smadi, Reliable numerical algorithm for handling fuzzy integral equations of second kind in Hilbert spaces. Filomat 33 (2019) 583-597.

[4] T. Allahviranloo and B. Ghanbari, On the fuzzy fractional differential equation with interval Atangana-Baleanu fractional derivative approach. Chaos Solitons Fract. 130 (2020) 109397.

[5] T. Allahviranloo, S. Salahshour and S. Abbasbandy, Explicit solutions of fractional differential equations with uncertainty. Soft Comput. 16 (2012) 297-302.

[6] G.A. Anastassiou, Fuzzy mathematics: approximation theory. Vol. 251 of Studies in Fuzziness and Soft Computing. Springer, Berlin, Heidelberg (2010).

[7] M. Al-Smadi, Fractional residual series for conformable time-fractional Sawada-Kotera-Ito, Lax, and Kaup-Kupershmidt equations of seventh-order. Math. Methods Appl. Sci. (2021). DOI: 10.1002/mma.7507.

[8] M. Al-Smadi, Simplified iterative reproducing kernel method for handling time-fractional BVPs with error estimation. Ain Shams Eng. J. 9 (2018) 2517-2525.

[9] M. Al-Smadi and O. Abu Arqub, Computational algorithm for solving Fredholm time-fractional partial integrodifferential equations of Dirichlet functions type with error estimates. Appl. Math. Comput. 342 (2019) 280-294.

[10] M. Al-Smadi, O. Abu Arqub and S. Momani, A computational method for two-point boundary value problems of fourth-order mixed integrodifferential equations. Math. Probl. Eng. 2013 (2013) 832074.

[11] M. Al-Smadi, O. Abu Arqub and D. Zeidan, Fuzzy fractional differential equations under the Mittag-Leffler kernel differential operator of the ABC approach: theorems and applications. Chaos Solitons Fract. 146 (2021) 110891.

[12] M. Al-Smadi, O. Abu Arqub and S. Hadid, Approximate solutions of nonlinear fractional Kundu-Eckhaus and coupled fractional massive Thirring equations emerging in quantum field theory using conformable residual power series method. Phys. Scr. 95 (2020) 105205.

[13] M. Al-Smadi, O. Abu Arqub and S. Hadid, An attractive analytical technique for coupled system of fractional partial differential equations in shallow water waves with conformable derivative. Commun. Theor. Phys. 72 (2020) 085001.

[14] M. Al-Smadi, O. Abu Arqub and M. Gaith, Numerical simulation of telegraph and Cattaneo fractional-type models using adaptive reproducing kernel framework. Math. Methods Appl. Sci. (2020). DOI: 10.1002/mma.6998.

[15] M. Al-Smadi, O. Abu Arqub and S. Momani, Numerical computations of coupled fractional resonant Schrödinger equations arising in quantum mechanics under conformable fractional derivative sense. Phys. Scr. 95 (2020) 075218.

[16] M. Al-Smadi, O. Abu Arqub, N. Shawagfeh and S. Momani, Numerical investigations for systems of second-order periodic boundary value problems using reproducing kernel method. Appl. Math. Comput. 291 (2016) 137-148.

[17] M. Al-Smadi, A. Freihat, H. Khalil, S. Momani, R.A. Khan, Numerical multistep approach for solving fractional partial differential equations. Int. J. Comput. Methods 14 (2017) 1750029.

[18] N. Aronszajn, Theory of reproducing kernels. Trans. Am. Math. Soc. 68 (1950) 337-404.

[19] A. Atangana, On the new fractional derivative and application to nonlinear Fisher's reaction-diffusion equation. Appl. Math. Comput. 273 (2016) 948-956.

[20] A. Atangana and D. Baleanu, Nonlinear fractional Jaulent-Miodek and Whitham-Broer-Kaup equations within Sumudu transform. Abstr. Appl. Anal. 2013 (2013) 160681.

[21] A. Atangana and D. Baleanu, New fractional derivatives with non-local and non-singular kernel: theory and application to heat transfer model. Thermal Sci. 20 (2016) 763-769.

[22] A. Atangana, J.F. Gómez-Aguilar, M.O. Kolade and J.Y. Hristov, Fractional differential and integral operators with nonsingular and non-local kernel with application to nonlinear dynamical systems. Chaos Solitons Fract. 132 (2020) 109493.

[23] D. Baleanu and A. Fernandez, On some new properties of fractional derivatives with Mittag-Leffler kernel. Commun. Nonlinear Sci. Numer. Simul. 59 (2018) 444-462.

[24] B. Bede and L. Stefanini, Generalized differentiability of fuzzy valued functions. Fuzzy Sets Syst. 230 (2013) $119-141$.

[25] M. Caputo and M. Fabrizio, A new definition of fractional derivative without singular Kernel. Progr. Fract. Differ. Appl. 1 (2015) 73-85.

[26] M. Cui and Y. Lin, Nonlinear Numerical Analysis in the Reproducing Kernel Space. Nova Science, New York, NY, USA (2009). 
[27] P. Diamond and P. Kloeden, Towards the theory of fuzzy differential equations. Fuzzy Sets Syst. 100 (1999) 63-71.

[28] N. Djeddi, S. Hasan, M. Al-Smadi and S. Momani, Modified analytical approach for generalized quadratic and cubic logistic models with Caputo-Fabrizio fractional derivative. Alexandria Eng. J. 59 (2020) 5111-5122.

[29] D. Dubois and H. Prade, Operations on fuzzy numbers. Int. J. Syst. Sci. 9 (1978) 613-626.

[30] D. Dubois and H. Prade, Towards fuzzy differential calculus: Part 3. Differentiation, Fuzzy Sets Syst. 8 (1982) $225-233$.

[31] H. Dutta, A. Akdemir and A. Atangana, Fractional order analysis: theory, methods and applications. John Wiley and Sons Ltd, Hoboken, USA (2020).

[32] M. Friedman, M. Ma and A. Kandel, Numerical solutions of fuzzy differential and integral equations. Fuzzy Sets Syst. 106 (1999) 35-48.

[33] R. Goetschel and W. Voxman, Elementary fuzzy calculus. Fuzzy Sets Syst. 18 (1986) 31-43.

[34] H. Günerhan, H. Dutta, M.A. Dokuyucu and W. Adel, Analysis of a fractional HIV model with Caputo and constant proportional Caputo operators. Chaos Solitons Fract. 139 (2020) 110053.

[35] N. Harrouche, S. Momani, S. Hasan and M. Al-Smadi, Computational algorithm for solving drug pharmacokinetic model under uncertainty with nonsingular kernel type Caputo-Fabrizio fractional derivative. Alexandria Eng. J. 60 (2021) 4347-4362.

[36] S. Hasan, M. Al-Smadi, A. El-Ajou, S. Momani, S. Hadid and Z. Al-Zhour, Numerical approach in the Hilbert space to solve a fuzzy Atangana-Baleanu fractional hybrid system. Chaos Solitons Fract. 143 (2021) 110506.

[37] S. Hasan, M. Al-Smadi, A. Freihet and S. Momani, Two computational approaches for solving a fractional obstacle system in Hilbert space. Adv. Differ. Equ. 2019 (2019) 55.

[38] S. Hasan, A. El-Ajou, S. Hadid, M. Al-Smadi and S. Momani, Atangana-Baleanu fractional framework of reproducing kernel technique in solving fractional population dynamics system. Chaos Solitons Fract. 133 (2019) 109624.

[39] J. Hristov, Response functions in linear viscoelastic constitutive equations and related fractional operators, Math. Model. Nat. Phenom. 14 (2019) 305.

[40] O. Kaleva, Fuzzy differential equations. Fuzzy Sets Syst. 24 (1987) 301-317.

[41] A. Kandel and W. Byatt, Fuzzy differential equations. Proc. Int. Conf. Cybern. Soc. (1978) 1213-1216.

[42] A.A. Kilbas, H.M. Srivastava and J.J. Trujillo, Theory and applications of fractional differential equations. Vol. 204 of em North-Holland Mathematics Studies. Elsevier Science (2006).

[43] S. Kumar, A. Kumar, B. Samet and H. Dutta, A study on fractional host-parasitoid population dynamical model to describe insect species. Numer. Methods Partial Differ. Equ. 37 (2021) 1673-1692.

[44] D. Kumar, J. Singh and D. Baleanu, On the analysis of vibration equation involving a fractional derivative with Mittag-Leffler law. Math. Methods Appl. Sci. 43 (2020) 443-457.

[45] C.C. Lee, Fuzzy logic in control systems. Fuzzy Logic Control. 20 (1990) 404-418.

[46] S. Momani, O. Abu Arqub, A. Freihat and M. Al-Smadi, Analytical approximations for Fokker-Planck equations of fractional order in multistep schemes. Appl. Comput. Math. 15 (2016) 319-330.

[47] M. Puri and D. Ralescu, Fuzzy random variables. J. Math. Anal. Appl. 114 (1986) 409-422.

[48] S. Salahshour, T. Allahviranloo, S. Abbasbandy and D. Baleanu, Existence and uniqueness results for fractional differential equations with uncertainty. Adv. Differ. Equ. 2012 (2012) 112.

[49] J. Singh, A. Ahmadian, S. Rathore, D. Kumar, D. Baleanu, M. Salimi and S. Salahshour, An efficient computational approach for local fractional poisson equation in fractal media. Numer. Methods Partial Differ. Equ. 37 (2021) 1439-1448.

[50] J. Singh, H.K. Jassim and D. Kumar, An efficient computational technique for local fractional Fokker Planck equation. Physica A 555 (2020) 124525.

[51] M. Yavuz, Characterizations of two different fractional operators without singular kernel. Math. Model. Nat. Phenom. 14 (2019) 302

[52] M. Yavuz, Fundamental solutions to the Cauchy and Dirichlet problems for a heat conduction equation equipped with the Caputo-Fabrizio differentiation. Heat Conduct. 2019 (2019) 95-107.

[53] M. Yavuz, European option pricing models described by fractional operators with classical and generalized Mittag-Leffler kernels. Numer. Methods Partial Differ. Equ. (2020). https://doi.org/10.1002/num.22645.

[54] M. Yavuz and T. Abdeljawad, Nonlinear regularized long-wave models with a new integral transformation applied to the fractional derivative with power and Mittag-Leffler kernel. Adv. Differ. Equ. 2020 (2020) 367.

[55] L. Zadeh, Fuzzy sets. Inf. Control 8 (1965) 338-353.

[56] J. Zhang, G. Wang, X. Zhi and C. Zhou, Generalized Euler-Lagrange equations for fuzzy fractional variational problems under gH-Atangana-Baleanu differentiability. J. Funct. Spaces 2018 (2018) 2740678. 